\title{
Stability and Permanence of a Pest Management Model with Impulsive Releasing and Harvesting
}

\author{
Jiangtao Yang and Zhichun Yang \\ Department of Mathematics, Chongqing Normal University, Chongqing 400047, China \\ Correspondence should be addressed to Zhichun Yang; zhichy@yahoo.com.cn
}

Received 16 January 2013; Accepted 27 February 2013

Academic Editor: Chuangxia Huang

Copyright ( 2013 J. Yang and Z. Yang. This is an open access article distributed under the Creative Commons Attribution License, which permits unrestricted use, distribution, and reproduction in any medium, provided the original work is properly cited.

\begin{abstract}
We formulate a pest management model with periodically releasing infective pests, immature and mature natural enemies, and harvesting pests and crops at two different fixed moments. Sufficient conditions ensuring the locally and globally asymptotical stability of the susceptible pest-eradication period solution are found by means of Floquet theory, small amplitude perturbation techniques, and multicomparison results. Furthermore, the permanence of system is also derived. By numerical analysis, we also show that impulsive releasing and harvesting at two different fixed moments can bring obvious effects on the dynamics of system, which also corroborates our theoretical results.
\end{abstract}

\section{Introduction}

As is known to all, pest outbreaks often cause serious ecological and economic problems. Therefore, how to effectively control insects and other arthropods has become an increasingly complex issue. Usually, chemical pesticides were taken as a relatively simple way to solve the pest-related problems, and some mathematical models on pest management with toxin (pesticide) input were studied in [1-4]. However, the overuse of chemical pesticides may create new ecological and sociological harm such as pesticide pollution and pesticideresistant pest varieties and inflicts harmful effects on humans and so forth. Therefore, nonchemical use instead for pest control has become a hot topic in order to reduce pest density to tolerable levels and minimize the damage caused. For instance, biological control methods by periodically releasing infective pests or their natural enemies are often taken due to their advantage in the aspects of self-sustainable mechanism, lower environmental impact, and cost effectiveness.

Recently, some biocontrol models on pest management described by impulsive differential equation were proposed and the dynamics such as stability, permanence, periodicity, and bifurcation are deeply investigated (see also, e.g., [2-12]). In [5], an impulsive system to model the process of periodic releasing natural enemies and harvesting pest at different fixed time for pest control is considered, and the sufficient conditions on the existence and global stability of the periodic solution are derived for the given model. Georgescu et al. $[6,7]$ construct an integrated pest management model which relies on the simultaneous periodic release of infective pest individuals and of natural predators with age structure and obtain some sufficient conditions on the local and global stability, permanence, and bifurcation of the systems. However, most of the existing models on pest management scarcely take into account the factor on the relation between pest and its food (e.g., crop). In fact, farmers may harvest crops several times in process of its growth, which should cause a great impact on the density of the pest.

Motived by the above discussion, we construct a model of pest control by periodically releasing infective pests, immature and mature natural enemies, and harvesting pests and crops. To account for the discontinuity of release and harvest at different fixed moments, our model is based on impulsive differential equations. We analyze the dynamical behavior of the system by using the theory of impulsive differential equation introduced in [13-15].

The rest of this paper is organized as follows. A pest management model with impulsive releasing and harvesting is introduced in Section 2 and some useful preliminaries are given in Section 3. Section 4 deals with stability and permanence analysis of system. In this section, two sufficient conditions are deduced including the locally and globally asymptotical stability of the susceptible pest-eradication 
period solution, the permanence of system is also discussed. A simple example and conclusions are given in Section 5.

\section{Model Description}

In the following, to establish our pest management model, we rely on the following biological assumptions.

(A1) The pest population is divided into two classes, the susceptible and infective. The infective pests neither recover nor reproduce and infective pests cannot damage crops. The disease is transmitted from infective pests to susceptible pests and does not propagate to predators.

(A2) In the absence of susceptible pests, the crops have a logistic growth rate with intrinsic birth rate $r$ and carrying capacity $K$.

(A3) The predators (natural enemies) have an age structure, that is, immature and mature. Only the mature predators have the ability to feed on susceptible pests, but do not prey on infective pests and crops.

(A4) The functional response of the susceptible pest is described by the abstract function $P_{1}$, the functional response of the mature predator is described by the abstract function $P_{2}$, and the infection rate is described by the abstract function $g$, where $P_{1}, P_{2}$, and $g$ satisfy certain assumptions outlined below.

On the basis of the above assumptions, we establish the following impulsively controlled system:

$$
\begin{aligned}
x^{\prime}(t)= & r x(t)\left(1-\frac{x(t)}{K}\right)-P_{1}(x(t)) S(t), \\
S^{\prime}(t)= & \beta P_{1}(x(t)) S(t)-g(I(t)) S(t) \\
& -P_{2}(S(t)) y_{M}(t)-d_{S} S(t), \\
I^{\prime}(t)= & g(I(t)) S(t)-d_{I} I(t), \\
y_{J}^{\prime}(t)= & \lambda P_{2}(S(t)) y_{M}(t)-d_{J} y_{J}(t)-m y_{J}(t), \\
y_{M}^{\prime}(t)= & m y_{J}(t)-d_{M} y_{M}(t), \\
t \neq(n+\tau-1) T, & t \neq n T,
\end{aligned}
$$

$$
\begin{aligned}
& \Delta x(t)=-\delta x(t), \\
& \Delta S(t)=-P_{S} S(t), \\
& \Delta I(t)=-P_{I} I(t), \\
& \Delta y_{J}(t)=-P_{J} y_{J}(t) \text {, } \\
& \Delta y_{M}(t)=-P_{M} y_{M}(t), \\
& t=(n+\tau-1) T,
\end{aligned}
$$

$$
\begin{aligned}
& \Delta x(t)=0, \\
& \Delta S(t)=0, \\
& \Delta I(t)=\delta_{I}, \\
& \Delta y_{J}(t)=\delta_{J}, \\
& \Delta y_{M}(t)=\delta_{M}, \\
& t=n T, n \in \mathbb{N},
\end{aligned}
$$

where $x(t)$ represents the density of the crop at time $t, S(t)$ represents the density of the susceptible pest at time $t, I(t)$ represents the density of the infective pest at time $t, y_{J}(t)$ and $y_{M}(t)$ represent the density of the immature and mature predator at time $t$, respectively; $r$ is the logistic intrinsic growth rate of the crop in the absence of the susceptible pest, $K$ is its carrying capacity; $0<\beta, \lambda \leq 1$ represent the conversion rate at which ingested preys in excess of what is needed for maintenance is translated into predator population increase; $m$ is the rate at which the immature predators become the mature predators. $d_{S}, d_{I}, d_{J}, d_{M}>0$ are the death rates of the susceptible pest population, infective pest population, and of the immature and mature predator population, respectively; $\Delta x(t)=x\left(t^{+}\right)-x(t), \Delta S(t)=$ $S\left(t^{+}\right)-S(t), \Delta I(t)=I\left(t^{+}\right)-I(t), \Delta y_{J}(t)=y_{J}\left(t^{+}\right)-y_{J}(t)$, $\Delta y_{M}(t)=y_{M}\left(t^{+}\right)-y_{M}(t) ; T$ is the period of the impulsive effect; $\delta\left(0 \leq \delta<\left(1-e^{-r T}\right) / 2\right)$ is the harvesting rate of crop population; $0 \leq P_{S}, P_{I}, P_{J}, P_{M}<1$ denote the transfer rate of susceptible pest population, infective pest population, immature and mature predator population at every impulsive period $(n+\tau-1) T(n \in \mathbb{N}, 0<\tau<1)$, respectively; $\delta_{I}, \delta_{J}$, $\delta_{M}>0$ represents the amount of infective pests, immature and mature predators, respectively, which are released at every impulsive period $n T(n \in \mathbb{N})$, respectively; Also, $P_{1}(\cdot)$, $P_{2}(\cdot), g(\cdot) \in H$, here $H=\left\{f: R \rightarrow R \mid f(0)=0, f^{\prime}(x)>0\right.$ and $f^{\prime \prime}(x) \leq 0$ for all $\left.x>0\right\}$.

Some familiar examples of functions $f \in H$ in the biological literature include

$$
\begin{aligned}
& \text { (F1) } f_{1}(x)=a x \text {, with } a>0 ; \\
& \text { (F2) } f_{2}(x)=a x /(1+b x) \text {, with } a, b>0 ; \\
& \text { (F3) } f_{3}(x)=a\left(1-e^{-b x}\right) \text {, with } a, b>0,
\end{aligned}
$$

where functions $(F 1)$ and $(F 2)$ are known as Holling type functional responses (see, [16-26]), and (F3) belongs to Ivlev type functional responses (see, [27-30]).

\section{Preliminaries}

In this section, we will give some definitions and lemmas, which will be useful for our main results. Let $\mathbb{R}_{+}=$ $[0, \infty)$ and $\mathbb{R}_{+}^{5}=\left\{X=\left(x(t), S(t), I(t), y_{J}(t), y_{M}(t)\right) \in\right.$ $\left.\mathbb{R}^{5} \mid x(t), S(t), I(t), y_{J}(t), y_{M}(t) \geq 0\right\}$. Denote $f=$ $\left(f_{x}, f_{S}, f_{I}, f_{J}, f_{M}\right)^{T}$ the map defined by the right hand of the first five equations in system (1). Let $V: \mathbb{R}_{+} \times \mathbb{R}_{+}^{5} \rightarrow \mathbb{R}_{+}$, then $V \in V_{0}$ if 
(1) $V$ is continuous in $((n-1) T,(n+\tau-1) T] \times \mathbb{R}_{+}^{5}$, $((n+\tau-1) T, n T] \times \mathbb{R}_{+}^{5}$ and for each $x \in R_{+}^{5}, n \in \mathbb{N}$, $\lim _{(t, y) \rightarrow\left((n+\tau-1) T^{+}, x\right)} V(t, y)=V\left((n+\tau-1) T^{+}, x\right)$ and $\lim _{(t, y) \rightarrow\left(n T^{+}, x\right)} V(t, y)=V\left(n T^{+}, x\right)$ exist.

(2) $V$ is locally Lipschitzian $x$.

Definition 1. Letting $V \in V_{0}$, one defines the upper right derivative of $V$ with respect to the impulsive differential system $(1)$ at $(t, x) \in((n-1) T,(n+\tau-1) T] \times \mathbb{R}_{+}^{5}$ and $((n+\tau-1) T, n T] \times \mathbb{R}_{+}^{5}$ by

$$
D^{+} V(t, x)=\limsup _{h \rightarrow 0^{+}} \frac{1}{h}[V(t+h, x+h f(t, x))-V(t, x)] .
$$

Definition 2. The system (1) is said to be permanent if there are positive constants $m, M>0$ and a finite time $T_{0}$ such that all solutions of (1) with initial values $x\left(0^{+}\right), S\left(0^{+}\right), I\left(0^{+}\right)$, $y_{J}\left(0^{+}\right), y_{M}\left(0^{+}\right), m \leq x(t), S(t), I(t), y_{J}(t), y_{M}(t) \leq M$ hold for all $t \geq T_{0}$, where $m$ and $M$ are independent of initial value, $T_{0}$ may depend on initial value.

Remark 3. The global existence and uniqueness of system (1) is guaranteed by the smoothness properties of $f$ (for details, see $[13,14])$.

Lemma 4 (see $[15]$ ). Let $V: \mathbb{R}_{+} \times \mathbb{R}^{n} \rightarrow \mathbb{R}_{+}^{m}$ satisfy $V_{i} \in$ $V_{0}, i=1,2, \ldots, m$, and assume that

$$
\begin{gathered}
D^{+} V(t, x(t)) \leq(\geq) g(t, V(t, x(t))), \quad t \neq(k+\tau-1) T, k T, \\
V\left(t, x\left(t^{+}\right)\right) \leq(\geq) \psi_{k}^{\tau}(V(t, x(t))), \quad t=(k+\tau-1) T, \\
V\left(t, x\left(t^{+}\right)\right) \leq(\geq) \psi_{k}(V(t, x(t))), \quad t=k T, k \in \mathbb{N}, \\
x\left(0^{+}\right)=x_{0},
\end{gathered}
$$

where $g: \mathbb{R}_{+} \times \mathbb{R}_{+}^{m} \rightarrow \mathbb{R}_{+}^{m}$ is continuous in $((k-1) T,(k+$ $\tau-1) T] \times \mathbb{R}^{m}$ and $((k+\tau-1) T, k T] \times \mathbb{R}^{m}$, for each $p \in$ $\mathbb{R}^{m}, k=1,2, \ldots$, the limit $\lim _{(t, q) \rightarrow\left((k+\tau-1) T^{+}, p\right)} g(t, q)=g((k+$ $\left.\tau-1) T^{+}, p\right)$ and $\lim _{(t, q) \rightarrow\left((k-1) T^{+}, p\right)} g(t, q)=g\left((k-1) T^{+}, p\right)$ exists. $g(t, q)$ is quasimonotone nondecreasing in $q . \psi_{k}^{\tau}, \psi_{k}$ : $\mathbb{R}_{+}^{m} \rightarrow \mathbb{R}_{+}^{m}$ is nondecreasing for all $k \in \mathbb{N}$. Let $\theta(t)$ be the maximal (minimal) solution of the following impulsive differential equation on $[0, \infty)$ :

$$
\begin{gathered}
w^{\prime}(t)=g(t, w(t)), \quad t \neq(k+\tau-1) T, k T, \\
w\left(t^{+}\right)=\psi_{k}^{\tau}(w(t)), \quad t=(k+\tau-1) T, \\
w\left(t^{+}\right)=\psi_{k}(w(t)), \quad t=k T, k \in \mathbb{N}, \\
w\left(0^{+}\right)=w_{0} .
\end{gathered}
$$

Then for any solution $x(t)$ of the system (3), $V\left(0^{+}\right.$, $\left.x_{0}\right) \leq(\geq) w_{0}$ implies that $V(t, x(t)) \leq(\geq) \theta(t)$ for all $t \geq 0$.
Lemma 5 (see $[13,15])$. Consider the following system:

$$
\begin{gathered}
v^{\prime}(t) \leq(\geq) p(t) v(t)+q(t), \quad t \neq t_{k}, \\
v\left(t_{k}^{+}\right) \leq(\geq) d_{k} v\left(t_{k}\right)+b_{k}, \quad t=t_{k}, k \in \mathbb{N}, \\
v\left(0^{+}\right) \leq(\geq) v_{0},
\end{gathered}
$$

where $p, q \in P C\left(\mathbb{R}_{+}, \mathbb{R}\right)$ and $d_{k} \geq 0, v_{0}$ and $b_{k}$ are constants. Suppose that

(A1) the sequence $t_{k}$ satisfies $0 \leq t_{1} \leq t_{2}<\cdots$, with $\lim _{t \rightarrow \infty} t_{k}=\infty$;

$(A 2) v \in P C^{\prime}\left(\mathbb{R}_{+}, \mathbb{R}\right)$ and $v(t)$ is left-continuous at $t_{k}, k \in$ $\mathbb{N}$.

Then, for $t>0$,

$$
\begin{aligned}
v(t) \leq & (\geq) v_{0} e^{\int_{0}^{t} p(s) d s} \prod_{0<t_{k}<t} d_{k} \\
& +\sum_{0<t_{k}<t}\left(\prod_{t_{k}<t_{j}<t} d_{j} e^{\int_{t_{k}}^{t} p(s) d s}\right) b_{k} \\
& +\int_{0}^{t}\left(\prod_{s<t_{k}<t} d_{k}\right) e^{\int_{s}^{t} p(\tau) d \tau} q(s) d s .
\end{aligned}
$$

Lemma 6. There exists a constant $M=\max \{(1 / \lambda)((L / d)+$ $\left.\left.\left(\rho e^{d T} /\left(e^{d T}-1\right)\right)\right), K\right\}>0$, such that $x(t), S(t), I(t), y_{J}(t)$, $y_{M}(t) \leq M$ for each solution of (1) with $t$ large enough.

Proof. Since $x^{\prime}(t) \leq r x(1-(x(t) / K))$, then $\left.x^{\prime}(t)\right|_{x(t)=K} \leq 0$, and $x\left((n+\tau-1) T^{+}\right) \leq x((n+\tau-1) T)(0<\delta<1)$, so $x(t) \leq K$ for $t$ large enough. Let us define $V(t) \in V_{0}$ by $V(t)=\lambda \beta x(t)+\lambda S(t)+\lambda I(t)+y_{J}(t)+y_{M}(t)$ and denote $d=\min \left\{d_{S}, d_{I}, d_{J}, d_{M}\right\}$. Then, it is obvious that

$$
\begin{array}{r}
\frac{d V(t)}{d t}+d V(t) \leq \lambda \beta(r+d) x(t)-\frac{\lambda \beta r x^{2}(t)}{K}, \\
t \neq(n+\tau-1) T, t \neq n T .
\end{array}
$$

Since the right-hand side (7) is bounded from above by $L=$ $K \lambda \beta(r+d)^{2} / 4 r$, it follows that

$$
\frac{d V(t)}{d t}+d V(t) \leq L, \quad t \neq(n+\tau-1) T, t \neq n T
$$

When $t=(n+\tau-1) T$ and $t=n T$, it is easy to obtain that

$$
\begin{gathered}
V\left((n+\tau-1) T^{+}\right) \leq V((n+\tau-1) T), \\
V\left(n T^{+}\right)=V(n T)+\left(\lambda \delta_{I}+\delta_{J}+\delta_{M}\right) .
\end{gathered}
$$


Then, by Lemma 5, we can obtain that

$$
\begin{aligned}
V(t) \leq & V(0) e^{-d t}+\int_{0}^{t} L e^{-d(t-s)} d s \\
& +\sum_{0<k T<t} \rho e^{-d(t-k T)} \longrightarrow \frac{L}{d}+\frac{\rho e^{d T}}{e^{d T}-1}, \quad t \longrightarrow \infty
\end{aligned}
$$

where $\rho=\lambda \delta_{I}+\delta_{J}+\delta_{M}$. So it follows that $V(t)$ is uniformly bounded on $[0, \infty)$. The proof is completed.

Lemma 7 (see [31]). Let one consider the following impulsive control subsystem:

$$
\begin{gathered}
x^{\prime}(t)=r x(t)\left(1-\frac{x(t)}{K}\right), \quad t \neq(n+\tau-1) T, \\
\Delta x(t)=-\delta x(t), t=(n+\tau-1) T .
\end{gathered}
$$

Suppose $\delta_{0}^{*}=1-e^{-r T}$. Then one has the following results.

(1) If $\delta>\delta_{0}^{*}$, then the trivial periodic solution of system (11) is locally asymptotically stable.

(2) If $\delta<\delta_{0}^{*}$, then the system (11) has a unique positive periodic solution $x^{*}(t)$, which is globally asymptotically stable, where

$$
\begin{gathered}
x^{*}(t)=\frac{K\left(1-\delta-e^{-r T}\right)}{1-\delta-e^{-r T}+\delta e^{-r(t-(n+\tau-1) T)}}, \\
t \in((n+\tau-1) T,(n+\tau) T], n \in \mathbb{N}, \\
x^{*}\left(0^{+}\right)=x^{*}\left(n T^{+}\right)=\frac{K\left((1-\delta) e^{r T}-1\right)}{\left(e^{r \tau T}-1\right)+(1-\delta)\left(e^{r T}-e^{r \tau T}\right)} .
\end{gathered}
$$

Remark 8. From Lemma 7, we have

(1a) if $\delta_{0}^{*}>2 \delta$, then $x^{*}(t)>K / 2$ for all $t \geq 0$;

(2a) if $t \in((n-1) T, n T], n \in \mathbb{N}$, then the periodic solution $x^{*}(t)$ can be rewritten in the form

$$
x^{*}(t)=\left\{\begin{array}{c}
\frac{K\left(1-\delta-e^{-r T}\right)}{1-\delta-e^{-r T}+\delta e^{-r(T-\tau T)} e^{-r(t-(n-1) T)}}, \\
t \in((n-1) T,(n+\tau-1) T], \\
\frac{K\left(1-\delta-e^{-r T}\right)}{1-\delta-e^{-r T}+\delta e^{-r(t-(n+\tau-1) T)}}, \\
t \in((n+\tau-1) T, n T], n \in \mathbb{N} .
\end{array}\right.
$$

Lemma 9. Let one consider the following impulsive control subsystem:

$$
\begin{gathered}
z^{\prime}(t)=a(t)-d z(t), \quad t \neq(n+\tau-1) T, t \neq n T, \\
\Delta z(t)=-p z(t), \quad t=(n+\tau-1) T, \\
\Delta z(t)=\delta, \quad t=n T, n \in \mathbb{N}, \\
z\left(0^{+}\right)=z_{0},
\end{gathered}
$$

where $a(t)$ is a T-periodic $P C\left(\mathbb{R}_{+}, \mathbb{R}\right)$ function. $p, d$ are the positive real constants and $p<1$. Then system (14) has a unique T-periodic solution $z^{*}(t)$, and for each solution $z(t)$ of $(14)$, $z(t) \rightarrow z^{*}(t)$ as $t \rightarrow \infty$, where

$$
\begin{gathered}
z^{*}(t)=e^{-d(t-(n-1) T)}\left(z^{*}\left(0^{+}\right)+\int_{0}^{t-(n-1) T} a(s) e^{d s} d s\right), \\
t \in((n-1) T,(n+\tau-1) T], \\
z^{*}(t)=e^{-d(t-(n-1) T)}\left(z^{*}\left(\tau T^{+}\right) e^{d \tau T}+\int_{\tau T}^{t-(n-1) T} a(s) e^{d s} d s\right), \\
t \in((n+\tau-1) T, n T],
\end{gathered}
$$

$z^{*}\left(0^{+}\right)$

$$
=\frac{\left[(1-p) \int_{0}^{\tau T} a(s) e^{d s} d s+\int_{\tau T}^{T} a(s) e^{d s} d s\right] e^{-d T}+\delta}{1-(1-p) e^{-d T}},
$$

$z^{*}\left(\tau T^{+}\right)$

$$
=\frac{(1-p)\left[\int_{0}^{\tau T} a(s) e^{d s} d s+e^{-d T} \int_{\tau T}^{T} a(s) e^{d s} d s+\delta\right] e^{-d \tau T}}{1-(1-p) e^{-d T}} .
$$

Proof. First, it is easy to obtain that

$$
\begin{gathered}
z(t)=e^{-d t}\left(z\left(0^{+}\right)+\int_{0}^{t} a(s) e^{d s} d s\right), \quad t \in(0, \tau T], \\
z(t)=e^{-d(t-\tau T)} z\left(\tau T^{+}\right)+e^{-d t} \int_{\tau T}^{t} a(s) e^{d s} d s, \quad t \in(\tau T, T] .
\end{gathered}
$$

Since the $T$-periodicity requirement, we have

$$
\begin{aligned}
& z^{*}\left(\tau T^{+}\right)=e^{-d \tau T}\left(z^{*}\left(0^{+}\right)+\int_{0}^{\tau T} a(s) e^{d s} d s\right)(1-p) \\
& z^{*}\left(0^{+}\right)=e^{-d(T-\tau T)} z^{*}\left(\tau T^{+}\right)+e^{-d T} \int_{\tau T}^{T} a(s) e^{d s} d s+\delta
\end{aligned}
$$


By (17), we can obtain that

$z^{*}\left(0^{+}\right)$

$$
=\frac{\left[(1-p) \int_{0}^{\tau T} a(s) e^{d s} d s+\int_{\tau T}^{T} a(s) e^{d s} d s\right] e^{-d T}+\delta}{1-(1-p) e^{-d T}},
$$

$z^{*}\left(\tau T^{+}\right)$

$$
=\frac{(1-p)\left[\int_{0}^{\tau T} a(s) e^{d s} d s+e^{-d T} \int_{\tau T}^{T} a(s) e^{d s} d s+\delta\right] e^{-d \tau T}}{1-(1-p) e^{-d T}} .
$$

So, we will obtain the $T$-periodic solution of (14):

$$
\begin{array}{r}
z^{*}(t)=e^{-d(t-(n-1) T)}\left(z^{*}\left(0^{+}\right)+\int_{0}^{t-(n-1) T} a(s) e^{d s} d s\right), \\
t \in((n-1) T,(n+\tau-1) T], \\
z^{*}(t)=e^{-d(t-(n-1) T)}\left(z^{*}\left(\tau T^{+}\right) e^{d \tau T}+\int_{\tau T}^{t-(n-1) T} a(s) e^{d s} d s\right), \\
t \in((n+\tau-1) T, n T] .
\end{array}
$$

Let $Z(t)=z(t)-z^{*}(t)$. Substituting $Z(t)$ into (14), we have

$$
\begin{gathered}
Z^{\prime}(t)=-d Z(t), \quad t \neq(n+\tau-1) T, \quad t \neq n T, \\
\Delta Z(t)=-p Z(t), \quad t=(n+\tau-1) T, \\
\Delta Z(t)=0, \quad t=n T, n \in \mathbb{N}, \\
Z\left(0^{+}\right)=z_{0}-z^{*}\left(0^{+}\right) .
\end{gathered}
$$

Then, $Z(t)=Z\left(0^{+}\right) e^{-d t} \prod_{0<(n+\tau-1) T<t}(1-p) \rightarrow 0$, as $t \rightarrow \infty$. The proof is completed.

\section{Main Results}

4.1. Local and Global Stability. In this section, we will study the existence and stability of the system (1) susceptible pesteradication periodic solution $\left(x^{*}(t), 0, I^{*}(t), y_{J}^{*}(t), y_{M}^{*}(t)\right)$. To this purpose, it is seen first that when $S(t)=0$, system (1) can be rewritten in the form

$$
\begin{aligned}
& x^{\prime}(t)=r x(t)\left(1-\frac{x(t)}{K}\right), \\
& I^{\prime}(t)=-d_{I} I(t), \\
& y_{J}^{\prime}(t)=-\left(d_{J}+m\right) y_{J}(t), \\
& y_{M}^{\prime}(t)=m y_{J}(t)-d_{M} y_{M}(t), \\
& \quad t \neq(n+\tau-1) T, \\
& \Delta x(t)=-\delta x(t), \\
& \Delta I(t)=-P_{I} I(t), \\
& \Delta y_{J}(t)=-P_{J} y_{J}(t), \\
& \Delta y_{M}(t)=-P_{M} y_{M}(t), \\
& \quad t=(n+\tau-1) T, \\
& \Delta x(t)=0, \\
& \Delta I(t)=\delta_{I}, \\
& \Delta y_{J}(t)=\delta_{J}, \\
& \Delta y_{M}(t)=\delta_{M}, \\
& t=n T,
\end{aligned}
$$

which describes the dynamics of system (1) in the absence of the susceptible pest population. So, when $t \in((n-$ 1)T, $n T](n \in \mathbb{N})$, we can calculate the $T$-periodic solution of (21) by Lemmas 7 and 9 . It is seen that

$$
\begin{gathered}
x^{*}(t)=\left\{\begin{array}{c}
\frac{K\left(1-\delta-e^{-r T}\right)}{1-\delta-e^{-r T}+\delta e^{-r(T-\tau T)} e^{-r(t-(n-1) T)},} \\
t \in((n-1) T,(n+\tau-1) T], \\
\frac{K\left(1-\delta-e^{-r T}\right)}{2}
\end{array}\right. \\
I^{*}(t)=\left\{\begin{array}{c}
\frac{\delta_{I} e^{-d_{I}(t-(n-1) T)}}{1-\left(1-e^{-r T}+\delta e^{-r(t-(n+\tau-1) T)}\right.} \\
t \in((n+\tau-1) T, n T] \\
t \in((n-1) T,(n+\tau-1) T], \\
\frac{\delta_{I}\left(1-P_{I}\right) e^{-d_{I}(t-(n-1) T)}}{1-\left(1-P_{I}\right) e^{-d_{I} T}} \\
t \in((n+\tau-1) T, n T],
\end{array}\right.
\end{gathered}
$$

$$
y_{J}^{*}(t)=\left\{\begin{array}{l}
\frac{\delta_{J} e^{-\left(m+d_{J}\right)(t-(n-1) T)}}{1-\left(1-P_{J}\right) e^{-\left(m+d_{J}\right) T}}, \\
t \in((n-1) T,(n+\tau-1) T], \\
\frac{\delta_{J}\left(1-P_{J}\right) e^{-\left(m+d_{J}\right)(t-(n-1) T)}}{1-\left(1-P_{J}\right) e^{-\left(m+d_{J}\right) T}}, \\
t \in((n+\tau-1) T, n T]
\end{array}\right.
$$




$$
\begin{aligned}
& y_{M}^{*}(t) \\
& =\left\{\begin{array}{c}
e^{-d_{M}(t-(n-1) T)}\left(y_{M}^{*}\left(0^{+}\right)+A(t-(n-1) T)\right), \\
t \in((n-1) T,(n+\tau-1) T], \\
e^{-d_{M}(t-(n-1) T)}\left(\begin{array}{c}
\left.y_{M}^{*}\left(\tau T^{+}\right) e^{d_{M} \tau T}+B(t-(n-1) T)\right), \\
t \in((n+\tau-1) T, n T],
\end{array}\right.
\end{array}\right. \\
& y_{M}^{*}\left(0^{+}\right)=\frac{\left[\left(1-P_{M}\right) A(\tau T)+B(T)\right] e^{-d_{M} T}+\delta_{M}}{1-\left(1-P_{M}\right) e^{-d_{M} T}}, \\
& y_{M}^{*}\left(\tau T^{+}\right)=\frac{\left(1-P_{M}\right)\left[A(\tau T)+e^{-d_{M} T} B(T)+\delta_{M}\right] e^{-d_{M} \tau T}}{1-\left(1-P_{M}\right) e^{-d_{M} T}},
\end{aligned}
$$

where

$$
\begin{array}{r}
A(t)=\frac{m \delta_{J}\left(e^{\left(d_{M}-\left(m+d_{J}\right)\right) t}-1\right)}{\left(1-\left(1-P_{J}\right) e^{-\left(m+d_{J}\right) T}\right)\left(d_{M}-\left(m+d_{J}\right)\right)}, \\
t \in(0, \tau T], \\
B(t)=\frac{m \delta_{J}\left(1-P_{J}\right)\left(e^{\left(d_{M}-\left(m+d_{J}\right)\right) t}-e^{\left(d_{M}-\left(m+d_{J}\right)\right) \tau T}\right)}{\left(1-\left(1-P_{J}\right) e^{-\left(m+d_{J}\right) T}\right)\left(d_{M}-\left(m+d_{J}\right)\right)}, \\
t \in(\tau T, T] .
\end{array}
$$

To discuss the locally asymptotical stability of the susceptible pest-eradication periodic solution, we now introduce the Floquet theory for a linear impulsive control system:

$$
\begin{gathered}
\omega^{\prime}(t)=A(t) \omega(t), \quad t \neq \tau_{k}, \\
\Delta \omega(t)=B_{k} \omega(t), \quad t=\tau_{k}, k \in \mathbb{N},
\end{gathered}
$$

under the following conditions:

$H 1: A(\cdot) \in \operatorname{PC}\left(\mathbb{R}, M_{n}(\mathbb{R})\right)$ and $A(t+T)=A(t)$ for $t \geq 0$.

H2: $B_{k} \in M_{n}$, $\operatorname{det}\left(I_{n}+B_{k}\right) \neq 0, \tau_{k}<\tau_{k+1}$ for $k \in \mathbb{N}$, and $I_{n}$ denotes the $n \times n$ real identity matrix.

H3: There is a $q \in \mathbb{N}$ such that $B_{k+q}=B_{k}, \tau_{k+q}=\tau_{k}+T$ for $k \in \mathbb{N}$.

Let $\Psi(t)$ be a fundamental matrix of (29), then there is a unique reversible matrix $M \in M_{n}(\mathbb{R})$ such that $\Psi(t+T)=$ $\Psi(t) M$ for all $t \in \mathbb{R}$, which is called the monodromy matrix of (29) corresponding to $\Psi$. All monodromy matrices of (29) are similar and they have the same eigenvalues $\lambda_{1}, \lambda_{2}, \ldots, \lambda_{n}$, which are called the Floquet multipliers of (29).

Lemma 10 (see [13] (Floquet theory)). Let the conditions H1H3 hold. Then system (29) have the following properties

(1) stable if and only if all Floquet multipliers $\lambda_{i}(1 \leq i \leq$ n) of (29) satisfy $\left|\lambda_{i}\right| \leq 1$ and moreover, to those $\lambda_{i}$ for which $\left|\lambda_{i}\right|=1$, there correspond simple elementary divisors;
(2) asymptotically stable if and only if all Floquet multipliers $\lambda_{i}(1 \leq i \leq n)$ of (29) satisfy $\left|\lambda_{i}\right|<1$;

(3) unstable if there is a Floquet multipliers $\lambda_{i}(1 \leq i \leq n)$ of (29) such that $\left|\lambda_{i}\right|>1$.

In the following, we present two main results with the locally and globally asymptotical stability of the susceptible pest-eradication periodic solution $\left(x^{*}(t), 0, I^{*}(t), P_{J}^{*}(t)\right.$, $\left.P_{M}^{*}(t)\right)$.

Theorem 11. If

$$
\begin{aligned}
& \beta \int_{0}^{T} P_{1}\left(x^{*}(t)\right) d t-\int_{0}^{T} g\left(I^{*}(t)\right) d t \\
& \quad-P_{2}^{\prime}(0) \int_{0}^{T} y_{M}^{*}(t) d t-d_{S} T<\ln \frac{1}{1-P_{S}},
\end{aligned}
$$

then the susceptible pest-eradication periodic solution $\left(x^{*}(t), 0\right.$, $\left.I^{*}(t), y_{J}^{*}(t), y_{M}^{*}(t)\right)$ of system (1) is locally asymptotically stable.

Proof. Let $\left(x(t), S(t), I(t), y_{J}(t), y_{M}(t)\right)$ be any solution of system (1). We define error $e_{1}(t)=x(t)-x^{*}(t), e_{2}(t)=$ $S(t), e_{3}(t)=I(t)-I^{*}(t), e_{4}(t)=y_{J}(t)-y_{J}^{*}(t), e_{5}(t)=$ $y_{M}(t)-y_{M}^{*}(t)$. The linearized system of $(1)$ at $\left(x^{*}(t)\right.$, $\left.0, I^{*}(t), y_{J}^{*}(t), y_{M}^{*}(t)\right)$ is

$$
\begin{aligned}
& e_{1}^{\prime}(t)=\left(r-2 r \frac{x^{*}(t)}{K}\right) e_{1}(t)-P_{1}\left(x^{*}(t)\right) e_{2}(t) \\
& e_{2}^{\prime}(t)=\left(\beta P_{1}\left(x^{*}(t)\right)-g\left(I^{*}(t)\right)-P_{2}^{\prime}(0) y_{M}^{*}(t)-d_{S}\right) e_{2}(t) \\
& e_{3}^{\prime}(t)=g\left(I^{*}(t)\right) e_{2}(t)-d_{I} e_{3}(t) \\
& e_{4}^{\prime}(t)=\lambda P_{2}^{\prime}(0) y_{M}^{*}(t) e_{2}(t)-\left(d_{J}+m\right) e_{4}(t), \\
& e_{5}^{\prime}(t)=m e_{4}(t)-d_{M} e_{5}(t)
\end{aligned}
$$

$$
t \neq(n+\tau-1) T
$$

$t \neq n T$,

$$
\begin{aligned}
& \Delta e_{1}(t)=-\delta e_{1}(t), \\
& \Delta e_{2}(t)=-P_{S} e_{2}(t), \\
& \Delta e_{3}(t)=-P_{I} e_{3}(t), \\
& \Delta e_{4}(t)=-P_{J} e_{4}(t), \\
& \Delta e_{5}(t)=-P_{M} e_{5}(t), \\
& t=(n+\tau-1) T, \\
& \Delta e_{1}(t)=\Delta e_{2}(t)=\Delta e_{3}(t)=\Delta e_{4}(t)=\Delta e_{5}(t)=0,
\end{aligned}
$$$$
t=n T .
$$

Let $\Psi(t)$ be the fundamental matrix of (31), then $\Psi(t)$ satisfies 


$$
\frac{d \Psi(t)}{d t}=\left(\begin{array}{ccccc}
r-2 r \frac{x^{*}(t)}{K} & P_{1}\left(x^{*}(t)\right) & 0 & 0 & 0 \\
0 & \beta P_{1}\left(x^{*}(t)\right)-g\left(I^{*}(t)\right)-P_{2}^{\prime}(0) y_{M}^{*}(t)-d_{S} & 0 & 0 & 0 \\
0 & g\left(I^{*}(t)\right) & -d_{I} & 0 & 0 \\
0 & \lambda P_{2}^{\prime}(0) y_{M}^{*}(t) & 0 & -\left(d_{J}+m\right) & 0
\end{array}\right) \Psi(t) .
$$

Then, a fundamental matrix $\Psi(t)\left(\Psi(0)=I_{4}\right)$ of (31) is

$$
\Psi(t)=\left(\begin{array}{ccccc}
e^{\int_{0}^{t}\left(r-2 r\left(x^{*}(s) / K\right)\right) d s} & \phi_{12}(t) & 0 & 0 & 0 \\
0 & e^{\int_{0}^{t}\left(\beta P_{1}\left(x^{*}(s)\right)-g\left(I^{*}(s)\right)-P_{2}^{\prime}(0) y_{M}^{*}(s)-d_{S}\right) d s} & 0 & 0 & 0 \\
0 & \phi_{32}(t) & e^{-d_{I} t} & 0 & 0 \\
0 & \phi_{42}(t) & 0 & e^{-\left(d_{J}+m\right) t} & 0 \\
0 & \phi_{52}(t) & 0 & \phi_{54}(t) & e^{-d_{M} t}
\end{array}\right),
$$

where

$$
\begin{aligned}
& \phi_{12}(t)=-e^{-\int_{0}^{t}\left(r-2 r\left(x^{*}(s) / K\right)\right) d s} \\
& \times \int_{0}^{t} P_{1}\left(x^{*}(s)\right) e^{\int_{0}^{s}\left(\beta P_{1}\left(x^{*}(\xi)\right)-g\left(I^{*}(\xi)\right)-P_{2}^{\prime}(0) y_{M}^{*}(\xi)-d_{S}\right) d \xi} \\
& \times e^{\int_{0}^{s}\left(r-2 r\left(x^{*}(\xi) / K\right)\right) d \xi} d s, \\
& \phi_{32}(t) \\
& =e^{-d_{I} t} \int_{0}^{t} g\left(I^{*}(s)\right) e^{\int_{0}^{s}\left(\beta P_{1}\left(x^{*}(\xi)\right)-g\left(I^{*}(\xi)\right)-P_{2}^{\prime}(0) y_{M}^{*}(\xi)-d_{S}\right) d \xi} \\
& \times e^{d_{I} s} d s \\
& \phi_{42}(t) \\
& =e^{-d_{J} t} \int_{0}^{t} \lambda P_{2}^{\prime}(0) y_{M}^{*}(s) e^{\int_{0}^{s}\left(\beta P_{1}\left(x^{*}(\xi)\right)-g\left(I^{*}(\xi)\right)-P_{2}^{\prime}(0) y_{M}^{*}(\xi)-d_{S}\right) d \xi} \\
& \times e^{d_{J} s} d s, \\
& \phi_{52}(t)=e^{-d_{M} t} \int_{0}^{t} m \phi_{42}(s) e^{d_{M} s} d s, \\
& \phi_{54}(t)=\frac{m\left(e^{-\left(d_{J}+m\right) t}-e^{-d_{M} t}\right)}{d_{M}-\left(d_{J}+m\right)} .
\end{aligned}
$$

The resetting impulsive condition of (31) becomes

$$
\begin{aligned}
& \left(\begin{array}{l}
e_{1}\left((n+\tau-1) T^{+}\right) \\
e_{2}\left((n+\tau-1) T^{+}\right) \\
e_{3}\left((n+\tau-1) T^{+}\right) \\
e_{4}\left((n+\tau-1) T^{+}\right) \\
e_{5}\left((n+\tau-1) T^{+}\right)
\end{array}\right) \\
& =\left(\begin{array}{ccccc}
1-\delta & 0 & 0 & 0 & 0 \\
0 & 1-P_{S} & 0 & 0 & 0 \\
0 & 0 & 1-P_{I} & 0 & 0 \\
0 & 0 & 0 & 1-P_{J} & 0 \\
0 & 0 & 0 & 0 & 1-P_{M}
\end{array}\right) \\
& \times\left(\begin{array}{c}
e_{1}((n+\tau-1) T) \\
e_{2}((n+\tau-1) T) \\
e_{3}((n+\tau-1) T) \\
e_{4}((n+\tau-1) T) \\
e_{5}((n+\tau-1) T)
\end{array}\right) \\
& \left(\begin{array}{l}
e_{1}\left(n T^{+}\right) \\
e_{2}\left(n T^{+}\right) \\
e_{3}\left(n T^{+}\right) \\
e_{4}\left(n T^{+}\right) \\
e_{5}\left(n T^{+}\right)
\end{array}\right)=\left(\begin{array}{lllll}
1 & 0 & 0 & 0 & 0 \\
0 & 1 & 0 & 0 & 0 \\
0 & 0 & 1 & 0 & 0 \\
0 & 0 & 0 & 1 & 0 \\
0 & 0 & 0 & 0 & 1
\end{array}\right)\left(\begin{array}{l}
e_{1}(n T) \\
e_{2}(n T) \\
e_{3}(n T) \\
e_{4}(n T) \\
e_{5}(n T)
\end{array}\right) .
\end{aligned}
$$

Then, it is easy to obtain all eigenvalues of

$$
M=\left(\begin{array}{ccccc}
1-\delta & 0 & 0 & 0 & 0 \\
0 & 1-P_{S} & 0 & 0 & 0 \\
0 & 0 & 1-P_{I} & 0 & 0 \\
0 & 0 & 0 & 1-P_{J} & 0 \\
0 & 0 & 0 & 0 & 1-P_{M}
\end{array}\right)
$$




$$
\times\left(\begin{array}{lllll}
1 & 0 & 0 & 0 & 0 \\
0 & 1 & 0 & 0 & 0 \\
0 & 0 & 1 & 0 & 0 \\
0 & 0 & 0 & 1 & 0 \\
0 & 0 & 0 & 0 & 1
\end{array}\right) \Psi(T)
$$

We have $\lambda_{1}=(1-\delta) e^{\int_{0}^{T}\left(r-2 r\left(x^{*}(s) / K\right)\right) d s}, \lambda_{2}=\left(1-P_{S}\right)$ $e^{\int_{0}^{T}\left(\beta P_{1}\left(x^{*}(s)\right)-g\left(I^{*}(s)\right)-P_{2}^{\prime}(0) y_{M}^{*}(s)-d_{S}\right) d s}, \lambda_{3}=\left(1-P_{I}\right) e^{-d_{I} T}<1$, $\lambda_{4}=\left(1-P_{J}\right) e^{-\left(d_{J}+m\right) T}<1$ and $\lambda_{5}=\left(1-P_{M}\right) e^{-d_{M} T}<1$. Since $x^{*}(t)>(K / 2)$, so $\lambda_{1}<1$. By the condition (30), we have $\lambda_{2}<$ 1. Therefore, according to Lemma 10, the susceptible pesteradication periodic solution $\left(x^{*}(t), 0, I^{*}(t), y_{J}^{*}(t), y_{M}^{*}(t)\right)$ of system (1) is locally asymptotically stable. The proof is completed.

Theorem 12. If

$$
\begin{aligned}
& \beta \int_{0}^{T} P_{1}\left(x^{*}(t)\right) d t-\int_{0}^{T} g\left(I^{*}(t)\right) d t \\
& \quad-\min _{0 \leq \omega \leq U_{S}} P_{2}^{\prime}(\omega) \int_{0}^{T} y_{M}^{*}(t) d t-d_{S} T<\ln \frac{1}{1-P_{S}},
\end{aligned}
$$

where $U_{S}$ is an ultimate boundedness constant for $S$, then the susceptible pest-eradication periodic solution $\left(x^{*}(t), 0\right.$, $\left.I^{*}(t), y_{J}^{*}(t), y_{M}^{*}(t)\right)$ of system (1) is globally asymptotically stable.

Proof. Since

$$
\begin{aligned}
& \beta \int_{0}^{T} P_{1}\left(x^{*}(t)\right) d t-\int_{0}^{T} g\left(I^{*}(t)\right) d t \\
& \quad-\min _{0 \leq \Phi \leq U_{S}} P_{2}^{\prime}(\omega) \int_{0}^{T} y_{M}^{*}(t) d t-d_{S} T<\ln \frac{1}{1-P_{S}},
\end{aligned}
$$

we can choose an $\varepsilon$ small enough such that

$$
\begin{aligned}
\beta \int_{0}^{T} & P_{1}\left(x^{*}(t)+\varepsilon\right) d t-\int_{0}^{T} g\left(I^{*}(t)-\varepsilon\right) d t \\
& -\min _{0 \leq \omega \leq U_{S}} P_{2}^{\prime}(\omega) \int_{0}^{T}\left(\overline{y_{M}^{*}(t)}-\varepsilon\right) d t-d_{S} T \\
& -\ln \frac{1}{1-P_{S}}=\epsilon<0,
\end{aligned}
$$

where $\overline{y_{M}^{*}(t)}$ is defined in the following. According to system (1), we have

$$
\begin{array}{r}
y_{M}^{\prime}(t)=m y_{J}(t)-d_{M} y_{M}(t), \\
t \neq(n+\tau-1) T, \\
t \neq n T,
\end{array}
$$

$$
\begin{aligned}
& \Delta x(t)=-\delta x(t), \\
& \Delta I(t)=-P_{I} I(t), \\
& \Delta y_{J}(t)=-P_{J} y_{J}(t), \\
& \Delta y_{M}(t)=-P_{M} y_{M}(t), \\
& \quad t=(n+\tau-1) T, \\
& \Delta x(t)=0, \\
& \Delta I(t)=\delta_{I}, \\
& \Delta y_{J}(t)=\delta_{J}, \\
& \Delta y_{M}(t)=\delta_{M}, \\
& t=n T, n \in \mathbb{N} .
\end{aligned}
$$

From the first equation of system (40), we obtain the following comparison system:

$$
\begin{gathered}
\nu^{\prime}(t)=r \nu(t)\left(1-\frac{\nu(t)}{K}\right), \quad t \neq(n+\tau-1) T, \\
\Delta \nu(t)=-\delta \nu(t), \quad t=(n+\tau-1) T .
\end{gathered}
$$

By Lemma 7, system (41) has a positive periodic solution $v^{*}(t)$, and for any solution $v(t)$ of $(41), v(t) \rightarrow v^{*}(t)$ as $t$ large enough, where $v^{*}(t)=x^{*}(t)$. Then, according to Lemmas 4 and 7 , there exists a positive constant $n^{*}$ such that for all $t \geq n^{*} T$

$$
x(t) \leq x^{*}(t)+\varepsilon .
$$

Let us define $V(t)=\left(V_{1}(t), V_{2}(t)\right)^{T} \in C\left[\mathbb{R}_{+} \times \mathbb{R}^{2}, \mathbb{R}_{+}^{2}\right]$ and $V_{i}(t) \in V_{0}, \quad(i=1,2)$, where $V_{1}(t)=I(t), V_{2}(t)=y_{J}(t)$. Then, we have

$$
\begin{aligned}
V^{\prime}(t) \geq\left(\begin{array}{c}
-d_{I} I(t) \\
-\left(d_{J}+m\right) y_{J}(t)
\end{array}\right)= & \left(\begin{array}{cc}
-d_{I} & 0 \\
0 & -\left(d_{J}+m\right)
\end{array}\right) V(t), \\
& t \neq(n+\tau-1) T, t \neq n T,
\end{aligned}
$$

$$
\begin{gathered}
V\left((n+\tau-1) T^{+}\right)=\left(\begin{array}{cc}
1-P_{I} & 0 \\
0 & 1-P_{J}
\end{array}\right) V((n+\tau-1) T), \\
V\left(n T^{+}\right)=\left(\begin{array}{ll}
1 & 0 \\
0 & 1
\end{array}\right) V(n T)+\left(\begin{array}{c}
\delta_{I} \\
\delta_{J}
\end{array}\right), \\
V\left(0^{+}\right)=\left(I\left(0^{+}\right), y_{J}\left(0^{+}\right)\right) .
\end{gathered}
$$


Then, the multicomparison system of (43) is

$$
\begin{gathered}
w^{\prime}(t)=A w(t), \quad t \neq(n+\tau-1) T, n T, \\
w\left(t^{+}\right)=B w(t), \quad t=(n+\tau-1) T, \\
w\left(t^{+}\right)=I_{2} w(t)+C, \quad t=n T, \\
w\left(0^{+}\right)=V\left(0^{+}\right),
\end{gathered}
$$

where $A=\left(\begin{array}{cc}-d_{I} & 0 \\ 0 & -\left(d_{I}+m\right)\end{array}\right), B=\left(\begin{array}{cc}1-P_{I} & 0 \\ 0 & 1-P_{J}\end{array}\right)$, and $C=\left(\begin{array}{l}\delta_{I} \\ \delta_{I}\end{array}\right)$.

By Lemma 9, it is easy to obtain a periodic solution $\left(I^{*}(t), y_{J}^{*}(t)\right)^{T}$ of system (45). Then, according to Lemmas 4 and 9 , one may find $n_{0}^{*}\left(n_{0}^{*}>n^{*}\right)$ such that for all $t \geq n_{0}^{*} T$

$$
I(t) \geq I^{*}(t)-\varepsilon, \quad y_{J}(t) \geq y_{J}^{*}(t)-\varepsilon .
$$

From the fourth equation of system (40), we have $y_{M}^{\prime}(t) \geq$ $m\left(y_{J}^{*}(t)-\varepsilon\right)-d_{M} y_{M}(t)$, by Lemmas 4 and 9 , there exists $n_{1}^{*}\left(n_{1}^{*}>n_{0}^{*}\right)$ such that for all $t \geq n_{1}^{*} T$

$$
y_{M}(t) \geq \overline{y_{M}^{*}(t)}-\varepsilon
$$

where

$$
\begin{aligned}
& \overline{y_{M}^{*}(t)}=\left\{\begin{array}{c}
e^{-d_{M}(t-(n-1) T)} \\
\times\left(\overline{y_{M}^{*}\left(0^{+}\right)}\right. \\
\left.\quad+m \int_{0}^{t-(n-1) T} \quad\left(y_{J}^{*}(t)-\varepsilon\right) e^{d_{M} s} d s\right), \\
\quad t \in((n-1) T,(n+\tau-1) T], \\
e^{-d_{M}(t-(n-1) T)} \\
\times\left(\overline{y_{M}^{*}\left(\tau T^{+}\right)} e^{d_{M} \tau T}\right. \\
\left.\quad+m \int_{\tau T}^{t-(n-1) T} \quad\left(y_{J}^{*}(t)-\varepsilon\right) e^{d_{M} s} d s\right), \\
\quad t \in((n+\tau-1) T, n T],
\end{array}\right. \\
& \overline{y_{M}^{*}\left(0^{+}\right)}=\left(m \left[\left(1-P_{M}\right) \int_{0}^{\tau T}\left(y_{J}^{*}(t)-\varepsilon\right) e^{d_{M} s} d s\right.\right. \\
& \left.\left.+\int_{\tau T}^{T}\left(y_{J}^{*}(t)-\varepsilon\right) e^{d_{M} s} d s\right] e^{-d_{M} T}+\delta_{M}\right) \\
& \times\left(1-\left(1-P_{M}\right) e^{-d_{M} T}\right)^{-1},
\end{aligned}
$$

$\overline{y_{M}^{*}\left(\tau T^{+}\right)}$

$$
\begin{aligned}
& =\left(( 1 - P _ { M } ) \left[m \int_{0}^{\tau T}\left(y_{J}^{*}(t)-\varepsilon\right) e^{d_{M} s} d s\right.\right. \\
& \left.\quad+m e^{-d_{M} T} \int_{\tau T}^{T}\left(y_{J}^{*}(t)-\varepsilon\right) e^{d_{M} s} d s+\delta_{M}\right] \\
& \left.\quad \times e^{-d_{M} \tau T}\right) \\
& \quad \times\left(1-\left(1-P_{M}\right) e^{-d_{M} T}\right)^{-1} .
\end{aligned}
$$

Therefore,

$$
\begin{gathered}
S^{\prime}(t) \leq\left[\beta P_{1}\left(x^{*}(t)+\varepsilon\right)-g\left(I^{*}(t)-\varepsilon\right)\right. \\
\left.-\min _{0 \leq \omega \leq U_{S}} P_{2}^{\prime}(\omega)\left(\overline{y_{M}^{*}(t)}-\varepsilon\right)-d_{S}\right] S(t), \\
t \neq(n+\tau-1) T, \\
t \neq n T, \\
\Delta S(t)=-P_{S} S(t), \quad t=(n+\tau-1) T, \\
\Delta S(t)=0, \quad t=n T,
\end{gathered}
$$

for $t \geq n_{1}^{*} T$. Let $N \in \mathbb{N}$ and $(N+\tau-1) \geq n_{1}^{*}$. Integrating (50) on $((n+\tau-1) T,(n+\tau) T], n \geq N$, we have

$$
\begin{aligned}
& S((n+\tau) T) \\
& \leq S((n+\tau-1) T)\left(1-P_{S}\right) \\
& \times e^{\int_{(n+\tau-1) T}^{(n+\tau) T}\left(\beta P_{1}\left(\widetilde{x^{*}(t)}-\varepsilon\right)-g\left(\widetilde{I^{*}(t)}+\varepsilon\right)-\min _{0 \leq \Delta \leq U_{S}} P_{2}^{\prime}(\varpi)\left(\widetilde{y_{M}^{*}(t)}+\varepsilon\right)-d_{S}\right) d t} \\
&= S((n+\tau-1) T) e^{\epsilon} .
\end{aligned}
$$

Then $S(t) \leq S((n+\tau) T) e^{k \epsilon}$ for $t \in((n+\tau+k) T,(n+\tau+k+1) T]$. Since $\epsilon<0$, we can easily get $S(t) \rightarrow 0$, as $t \rightarrow \infty$. In the following, we prove $x(t) \rightarrow x^{*}(t), I(t) \rightarrow I^{*}(t), y_{J}(t) \rightarrow$ $y_{J}^{*}(t), y_{M}(t) \rightarrow y_{M}^{*}(t)$, as $t \rightarrow \infty$. Give $\varepsilon_{0}>0$ small enough $\left(\varepsilon_{0}<\left(r / P_{1}^{\prime}(0)\right)\right)$, there must exist $n_{2}^{*}\left(n_{2}^{*}>n_{1}^{*}\right)$ such that $S(t)<\varepsilon_{0}$, for $t \geq n_{2}^{*} T$. Then, we have

$$
\begin{aligned}
& x^{\prime}(t) \geq\left(r-P_{1}^{\prime}(0) \varepsilon_{0}\right) x(t)\left(1-\frac{r x(t)}{K\left(r-P_{1}^{\prime}(0) \varepsilon_{0}\right)}\right), \\
& I^{\prime}(t) \leq-\left(d_{I}-g^{\prime}(0) \varepsilon_{0}\right) I(t), \\
& y_{J}^{\prime}(t) \leq \lambda P_{2}\left(\varepsilon_{0}\right) M-\left(d_{J}+m\right) y_{J}(t), \\
& y_{M}^{\prime}(t)=m y_{J}(t)-d_{M} y_{M}(t),
\end{aligned}
$$

$t \neq(n+\tau-1) T$

$t \neq n T$,

$$
\begin{aligned}
& \Delta x(t)=-\delta x(t), \\
& \Delta I(t)=-P_{I} I(t), \\
& \Delta y_{J}(t)=-P_{J} y_{J}(t), \\
& \Delta y_{M}(t)=-P_{M} y_{M}(t), \\
& t=(n+\tau-1) T, \\
& \Delta x(t)=0, \\
& \Delta I(t)=\delta_{I},
\end{aligned}
$$




$$
\begin{aligned}
\Delta y_{J}(t) & =\delta_{J}, \\
\Delta y_{M}(t) & =\delta_{M}, \\
t & =n T .
\end{aligned}
$$

Analyzing (52) with similarity as (40), there exists $n_{3}^{*}\left(n_{3}^{*}>\right.$ $n_{2}^{*}$ ) such that for all $t \geq n_{3}^{*} T$

$$
\begin{array}{cc}
x(t) \geq \widehat{x^{*}(t)}-\varepsilon, & I(t) \leq \widehat{I^{*}(t)}+\varepsilon, \\
y_{J}(t) \leq \widehat{y_{J}^{*}(t)}+\varepsilon, \quad y_{M}(t) \leq \widehat{y_{M}^{*}(t)}+\varepsilon,
\end{array}
$$

where

$$
\begin{aligned}
& \widehat{x^{*}(t)} \\
& =\left\{\begin{array}{c}
K\left(r-P_{1}^{\prime}(0) \varepsilon_{0}\right)\left(1-\delta-e^{-\left(r-P_{1}^{\prime}(0) \varepsilon_{0}\right) T}\right) \\
\times\left(r \left[1-\delta-e^{-\left(r-P_{1}^{\prime}(0) \varepsilon_{0}\right) T}\right.\right. \\
\left.\left.+\delta e^{-\left(r-P_{1}^{\prime}(0) \varepsilon_{0}\right)(T-\tau T)} e^{-\left(r-P_{1}^{\prime}(0) \varepsilon_{0}\right)(t-(n-1) T)}\right]\right)^{-1}, \\
t \in((n-1) T,(n+\tau-1) T], \\
K\left(r-P_{1}^{\prime}(0) \varepsilon_{0}\right)\left(1-\delta-e^{-\left(r-P_{1}^{\prime}(0) \varepsilon_{0}\right) T}\right) \\
\frac{r\left[1-\delta-e^{-\left(r-P_{1}^{\prime}(0) \varepsilon_{0}\right) T}+\delta e^{-\left(r-P_{1}^{\prime}(0) \varepsilon_{0}\right)(t-(n+\tau-1) T)}\right]}{t \in((n+\tau-1) T, n T],}
\end{array}\right.
\end{aligned}
$$

$\widehat{I^{*}(t)}$

$$
=\left\{\begin{array}{c}
\frac{\delta_{I} e^{-\left(d_{I}-g^{\prime}(0) \varepsilon_{0}\right)(t-(n-1) T)}}{1-\left(1-P_{I}\right) e^{-\left(d_{I}-g^{\prime}(0) \varepsilon_{0}\right) T}}, \\
t \in((n-1) T,(n+\tau-1) T], \\
\frac{\delta_{I}\left(1-P_{I}\right) e^{-\left(d_{I}-g^{\prime}(0) \varepsilon_{0}\right)(t-(n-1) T)}}{1-\left(1-P_{I}\right) e^{-\left(d_{I}-g^{\prime}(0) \varepsilon_{0}\right) T}}, \\
t \in((n+\tau-1) T, n T]
\end{array}\right.
$$

$\widehat{y_{J}^{*}(t)}$

$$
=\left\{\begin{array}{c}
e^{-\left(m+d_{J}\right)(t-(n-1) T)} \\
\times\left(\widehat{y_{J}^{*}\left(0^{+}\right)}+\lambda P_{2}\left(\varepsilon_{0}\right) M \int_{0}^{t-(n-1) T} e^{\left(m+d_{J}\right) s} d s\right), \\
t \in((n-1) T,(n+\tau-1) T], \\
e^{-\left(m+d_{J}\right)(t-(n-1) T)} \quad \\
\times\left(\widehat{y_{J}^{*}\left(\tau T^{+}\right) e^{\left(m+d_{J}\right) \tau T}}\right. \\
\left.+\lambda P_{2}\left(\varepsilon_{0}\right) M \int_{\tau T}^{t-(n-1) T} e^{\left(m+d_{J}\right) s} d s\right), \\
t \in((n+\tau-1) T, n T],
\end{array}\right.
$$

$\widehat{y_{J}^{*}\left(0^{+}\right)}$

$$
\begin{aligned}
=( & \lambda P_{2}\left(\varepsilon_{0}\right) M\left[\left(1-P_{J}\right) \int_{0}^{\tau T} e^{\left(m+d_{J}\right) s} d s+\int_{\tau T}^{T} e^{\left(m+d_{J}\right) s} d s\right] \\
& \left.\times e^{-\left(m+d_{J}\right) T}+\delta_{J}\right)\left(1-\left(1-P_{J}\right) e^{-\left(m+d_{J}\right) T}\right)^{-1}
\end{aligned}
$$

$$
\begin{aligned}
& \left.y_{J}^{*\left(\tau T^{+}\right.}\right) \\
& =\left(( 1 - P _ { J } ) \left[\lambda P_{2}\left(\varepsilon_{0}\right) M \int_{0}^{\tau T} e^{\left(m+d_{J}\right) s} d s\right.\right. \\
& \left.+e^{-\left(m+d_{J}\right) T} \lambda P_{2}\left(\varepsilon_{0}\right) M \int_{\tau T}^{T} e^{\left(m+d_{J}\right) s} d s+\delta_{J}\right] \\
& \left.\times e^{-\left(m+d_{J}\right) \tau T}\right)\left(1-\left(1-P_{J}\right) e^{-\left(m+d_{J}\right) T}\right)^{-1},
\end{aligned}
$$

$\widehat{y_{M}^{*}(t)}$

$$
\begin{aligned}
& \int \begin{array}{r}
e^{-d_{M}(t-(n-1) T)} \\
\times\left(\overline{y_{M}^{*}\left(0^{+}\right)}\right.
\end{array} \\
& \left.+m \int_{0}^{t-(n-1) T}\left(\widehat{y_{J}^{*}(t)}+\varepsilon\right) e^{d_{M^{s}}} d s\right) \\
& =\left\{\begin{array}{l}
e^{-d_{M}(t-(n-1) T)} \\
t \in((n-1) T,(n+\tau-1) T],
\end{array}\right. \\
& \times\left(y_{M}^{*}\left(\tau T^{+}\right) e^{d_{M} \tau T}\right. \\
& \left.+m \int_{\tau T}^{t-(n-1) T}\left(\widehat{y_{J}^{*}(t)}+\varepsilon\right) e^{d_{M} s} d s\right), \\
& t \in((n+\tau-1) T, n T],
\end{aligned}
$$

$\widehat{y_{M}^{*}\left(0^{+}\right)}$

$$
=\left(m \left[\left(1-P_{M}\right) \int_{0}^{\tau T}\left(\widehat{y_{J}^{*}(t)}+\varepsilon\right) e^{d_{M} s} d s\right.\right.
$$$$
\left.\left.+\int_{\tau T}^{T}\left(\widetilde{y_{J}^{*}(t)}+\varepsilon\right) e^{d_{M} s} d s\right] e^{-d_{M} T}+\delta_{M}\right)
$$$$
\times\left(1-\left(1-P_{M}\right) e^{-d_{M} T}\right)^{-1},
$$

$\widehat{y_{M}^{*}\left(\tau T^{+}\right)}$

$$
=\left(\left(1-P_{M}\right)\right.
$$

$$
\begin{aligned}
& \times\left[m \int_{0}^{\tau T}\left(\widehat{y_{J}^{*}(t)}+\varepsilon\right) e^{d_{M} s} d s+m e^{-d_{M} T}\right. \\
& \left.\left.\quad \times \int_{\tau T}^{T}\left(\widehat{y_{J}^{*}(t)}+\varepsilon\right) e^{d_{M} s} d s+\delta_{M}\right] e^{-d_{M} \tau T}\right) \\
& \times\left(1-\left(1-P_{M}\right) e^{-d_{M} T}\right)^{-1} .
\end{aligned}
$$

Letting $\varepsilon, \varepsilon_{0} \rightarrow 0$, we have $\overline{y_{M}^{*}(t)} \rightarrow y_{M}^{*}(t), \widehat{x^{*}(t)} \rightarrow x^{*}(t)$, $\widehat{I^{*}(t)} \rightarrow I^{*}(t), \widehat{y_{J}^{*}(t)} \rightarrow y_{J}^{*}(t), \widehat{y_{M}^{*}(t)} \rightarrow y_{M}^{*}(t)$. Together with (42), (46), (47), and (53), we get $x(t) \rightarrow x^{*}(t), I(t) \rightarrow$ $I^{*}(t), y_{J}(t) \rightarrow y_{J}^{*}(t), y_{M}(t) \rightarrow y_{M}^{*}(t)$ as $t \rightarrow \infty$. Therefore, the susceptible pest-eradication periodic solution $\left(x^{*}(t), 0, I^{*}(t), y_{J}^{*}(t), y_{M}^{*}(t)\right)$ is globally attractive. Since (37) implies (30), it follows from Theorem 11 that $\left(x^{*}(t), 0, I^{*}(t)\right.$, 
$\left.y_{J}^{*}(t)\right)$ is locally asymptotically stable. So, the susceptible pesteradication periodic solution $\left(x^{*}(t), 0, I^{*}(t), y_{J}^{*}(t), y_{M}^{*}(t)\right)$ of system (1) is globally asymptotically stable. The proof is completed.

4.2. Permanence. Next, we will discuss the permanence of system (1). In order to facilitate discussion, we give one lemma.

Lemma 13. There exists a constant $m_{4}>0$, such that $x(t)$, $I(t), y_{J}(t), y_{M}(t) \geq m_{4}$ for each solution of (1) with $t$ large enough.

Proof. First, we discuss $x(t)$. Since $S(t) \leq M$, by the first equation of system (1), we have

$$
\begin{gathered}
x^{\prime}(t) \geq\left(r-P_{1}^{\prime}(0) M\right) x(t)\left(1-\frac{r x(t)}{K\left(r-P_{1}^{\prime}(0) M\right)}\right), \\
t \neq(n+\tau-1) T, t \neq n T, \\
\Delta x(t)=-\delta x(t), \quad t=(n+\tau-1) T, \\
\Delta x(t)=0, \quad t=n T .
\end{gathered}
$$

Then, we obtain the following comparison system:

$$
\begin{gathered}
\chi^{\prime}(t)=\left(r-P_{1}^{\prime}(0) M\right) \chi(t)\left(1-\frac{r \chi(t)}{K\left(r-P_{1}^{\prime}(0) M\right)}\right), \\
t \neq(n+\tau-1) T, t \neq n T, \\
\Delta \chi(t)=-\delta \chi(t), \quad t=(n+\tau-1) T, \\
\Delta \chi(t)=0, \quad t=n T .
\end{gathered}
$$

Letting $r>P_{1}^{\prime}(0) M$ and $(1-\delta) e^{\left(r-P_{1}^{\prime}(0) M\right) T}>1$, by Lemma 7 , the system (56) has a positive periodic solution $\chi^{*}(t)$, and for any solution $\chi(t)$ of (56), $\chi(t) \rightarrow \chi^{*}(t)$ as $t$ large enough, where

$$
\chi^{*}(t)=\left\{\begin{array}{c}
K\left(r-P_{1}^{\prime}(0) M\right)\left(1-\delta-e^{-\left(r-P_{1}^{\prime}(0) M\right) T}\right) \\
\times\left(r \left[1-\delta-e^{-\left(r-P_{1}^{\prime}(0) M\right) T}\right.\right. \\
+\delta e^{-\left(r-P_{1}^{\prime}(0) M\right)(T-\tau T)} \\
\left.\left.\times e^{-\left(r-P_{1}^{\prime}(0) M\right)(t-(n-1) T)}\right]\right)^{-1}, \\
t \in((n-1) T,(n+\tau-1) T], \\
K\left(r-P_{1}^{\prime}(0) M\right)\left(1-\delta-e^{-\left(r-P_{1}^{\prime}(0) M\right) T}\right) \\
\times\left(r \left[1-\delta-e^{-\left(r-P_{1}^{\prime}(0) M\right) T}\right.\right. \\
\left.\left.+\delta e^{-\left(r-P_{1}^{\prime}(0) M\right)(t-(n+\tau-1) T)}\right]\right)^{-1}, \\
t \in((n+\tau-1) T, n T] .
\end{array}\right.
$$

According to Lemmas 4 and 7 , one may find $n_{4}^{*} \in \mathbb{N}$ such that $x(t) \geq \chi^{*}(t)-\varepsilon$ for $t \geq n_{4}^{*} T$. Since $\chi^{*}(t)-\varepsilon \geq(K(r-$ $\left.\left.P_{1}^{\prime}(0) M\right)\left(1-\delta-e^{-\left(r-P_{1}^{\prime}(0) M\right) T}\right) / r\left(1-e^{-\left(r-P_{1}^{\prime}(0) M\right) T}\right)\right)-\varepsilon=m_{0}>0$, so $x(t) \geq m_{0}$ for $t \geq n_{4}^{*} T$. Next, we will discuss the rest of parts.

From (46) and (47), we know that there exists $n_{5}^{*}\left(n_{5}^{*}>\right.$ $\left.\max \left\{n_{1}^{*}, n_{4}^{*}\right\}\right)$ such that $I(t) \geq I^{*}(t)-\varepsilon, y_{J}(t) \geq y_{J}^{*}(t)-\varepsilon$, and $y_{M}(t) \geq \overline{y_{M}^{*}(t)}-\varepsilon$ for all $t \geq n_{5}^{*} T$. By (22), (23), and (48), we have $I(t) \geq\left(\delta_{I}\left(1-P_{I}\right) e^{-d_{I} T} /\left(1-\left(1-P_{I}\right) e^{-d_{I} T}\right)\right)-\varepsilon=m_{1}>0$, $y_{J}(t) \geq\left(\delta_{J}\left(1-P_{J}\right) e^{-\left(m+d_{J}\right) T} /\left(1-\left(1-P_{J}\right) e^{-\left(m+d_{J}\right) T}\right)\right)-\varepsilon=m_{2}>$ 0 , and $y_{M}(t) \geq m_{M}-\varepsilon=m_{3}>0$, where $m_{M}=\min _{0<t \leq T} \overline{y_{M}^{*}(t)}$. Let $m_{4}=\min \left\{m_{0}, m_{1}, m_{2}, m_{3}\right\}$, then $x(t), I(t), y_{J}(t), y_{M}(t) \geq$ $m_{4}$ for $t \geq n_{5}^{*} T$. The proof is completed.

Theorem 14. If

$$
\begin{gathered}
\beta \int_{0}^{T} P_{1}\left(x^{*}(t)\right) d t-\int_{0}^{T} g\left(I^{*}(t)\right) d t-P_{2}^{\prime}(0) \\
\quad \times \int_{0}^{T} y_{M}^{*}(t) d t-d_{S} T>\ln \frac{1}{1-P_{S}}
\end{gathered}
$$

then system (1) is permanent.

Proof. By Lemmas 6 and 13, we have already known that there exist two constants $m_{4}, M>0$, such that $x(t), I(t), y_{J}(t), y_{M}(t) \geq m_{4}$ and $x(t), S(t), I(t), y_{J}(t), y_{M}(t) \leq$ $M$ for $t$ large enough. Thus, we only need to find $m^{*}>0$ such that $S(t) \geq m^{*}$ for $t$ large enough. We will do this in the following two steps.

Step 1. Let $m_{5}>0$ and $\varepsilon_{1}>0$ small enough, so that $m_{5}<$ $\min \left\{\left(r / P_{1}^{\prime}(0)\right),\left(d_{I} / g^{\prime}(0)\right), M\right\}$ and

$$
\begin{aligned}
& \beta \int_{0}^{T} P_{1}\left(\widetilde{x^{*}(t)}-\varepsilon_{1}\right) d t-\int_{0}^{T} g\left(\widetilde{I^{*}(t)}+\varepsilon_{1}\right) d t-P_{2}^{\prime}(0) \\
& \quad \times \int_{0}^{T}\left(\widetilde{y_{M}^{*}(t)}+\varepsilon_{1}\right) d t-d_{S} T-\ln \frac{1}{1-P_{S}}=\eta>0,
\end{aligned}
$$

where

$$
\widetilde{x^{*}(t)}=\left\{\begin{array}{c}
K\left(r-P_{1}^{\prime}(0) m_{5}\right)\left(1-\delta-e^{-\left(r-P_{1}^{\prime}(0) m_{5}\right) T}\right) \\
\times\left(r \left[\begin{array}{c}
1-\delta-e^{-\left(r-P^{\prime}{ }_{1}(0) m_{5}\right) T} \\
+\delta e^{-\left(r-P_{1}^{\prime}(0) m_{5}\right)(T-\tau T)} \\
\left.\left.\times e^{-\left(r-P_{1}^{\prime}(0) m_{5}\right)(t-(n-1) T)}\right]\right)^{-1}, \\
t \in((n-1) T,(n+\tau-1) T], \\
K\left(r-P_{1}^{\prime}(0) m_{5}\right)\left(1-\delta-e^{-\left(r-P_{1}^{\prime}(0) m_{5}\right) T}\right) \\
\times\left(r \left[1-\delta-e^{-\left(r-P_{1}^{\prime}(0) m_{5}\right) T}\right.\right. \\
\left.\left.+\delta e^{-\left(r-P_{1}^{\prime}(0) m_{5}\right)(t-(n+\tau-1) T)}\right]\right)^{-1}, \\
t \in((n+\tau-1) T, n T], \\
\frac{\delta_{I} e^{-\left(d_{I}-g^{\prime}(0) m_{5}\right)(t-(n-1) T)}}{1-\left(1-P_{I}\right) e^{-\left(d_{I}-g^{\prime}(0) m_{5}\right) T},} \\
t \in((n-1) T,(n+\tau-1) T], \\
\frac{\delta_{I}\left(1-P_{I}\right) e^{-\left(d_{I}-g^{\prime}(0) m_{5}\right)(t-(n-1) T)}}{1-\left(1-P_{I}\right) e^{-\left(d_{I}-g^{\prime}(0) m_{5}\right) T}} \\
t \in((n+\tau-1) T, n T],
\end{array}\right.\right.
\end{array}\right.
$$


$\widetilde{y_{J}^{*}(t)}$

$$
=\left\{\begin{array}{c}
e^{-\left(m+d_{J}\right)(t-(n-1) T)} \\
\times\left(\widetilde{y_{J}^{*}\left(0^{+}\right)}+\lambda P_{2}\left(m_{5}\right) M \int_{0}^{t-(n-1) T} e^{\left(m+d_{J}\right) s} d s\right), \\
e^{-\left(m+d_{J}\right)(t-(n-1) T)} \quad t \in((n-1) T,(n+\tau-1) T], \\
\times\left(y_{J}^{*\left(\tau T^{+}\right.}\right) e^{\left(m+d_{J}\right) \tau T} \\
\left.+\lambda P_{2}\left(m_{5}\right) M \int_{\tau T}^{t-(n-1) T} e^{\left(m+d_{J}\right) s} d s\right), \\
t \in((n+\tau-1) T, n T],
\end{array}\right.
$$$$
\widetilde{y_{J}^{*}\left(0^{+}\right)}
$$$$
=\left(\lambda P_{2}\left(m_{5}\right) M\left[\left(1-P_{J}\right) \int_{0}^{\tau T} e^{\left(m+d_{J}\right) s} d s+\int_{\tau T}^{T} e^{\left(m+d_{J}\right) s} d s\right]\right.
$$$$
\left.\times e^{-\left(m+d_{J}\right) T}+\delta_{J}\right)\left(1-\left(1-P_{J}\right) e^{-\left(m+d_{J}\right) T}\right)^{-1},
$$

$\widetilde{y_{J}^{*}\left(\tau T^{+}\right)}$

$$
\begin{aligned}
& =\left(\left(1-P_{J}\right)\right. \\
& \times\left[\lambda P_{2}\left(m_{5}\right) M \int_{0}^{\tau T} e^{\left(m+d_{J}\right) s} d s+e^{-\left(m+d_{J}\right) T}\right. \\
& \left.\quad \times \lambda P_{2}\left(m_{5}\right) M \int_{\tau T}^{T} e^{\left(m+d_{J}\right) s} d s+\delta_{J}\right] \\
& \left.\times e^{-\left(m+d_{J}\right) \tau T}\right)\left(1-\left(1-P_{J}\right) e^{-\left(m+d_{J}\right) T}\right)^{-1}
\end{aligned}
$$

\section{$\widetilde{y_{M}^{*}(t)}$}

$$
\left\{\begin{array}{c}
e^{-d_{M}(t-(n-1) T)} \\
\times\left(\widetilde{y_{M}^{*}\left(0^{+}\right)}+m \int_{0}^{t-(n-1) T}\right. \\
\left.\quad \times\left(\widetilde{y_{J}^{*}(t)}+\varepsilon_{1}\right) e^{d_{M} s} d s\right), \\
\quad t \in((n-1) T,(n+\tau-1) T], \\
e^{-d_{M}(t-(n-1) T)} \\
\times\left(\widetilde{y_{M}^{*}\left(\tau T^{+}\right) e^{d_{M} \tau T}}\right. \\
\left.+m \int_{\tau T}^{t-(n-1) T}\left(\widetilde{y_{J}^{*}(t)}+\varepsilon_{1}\right) e^{d_{M} s} d s\right), \\
t \in((n+\tau-1) T, n T],
\end{array}\right.
$$

$$
\widetilde{y_{M}^{*}\left(0^{+}\right)}
$$

$$
\begin{gathered}
=\left(m \left[\left(1-P_{M}\right) \int_{0}^{\tau T}\left(\widetilde{y_{J}^{*}(t)}+\varepsilon_{1}\right) e^{d_{M} s} d s\right.\right. \\
\left.+\int_{\tau T}^{T}\left(\widetilde{y_{J}^{*}(t)}+\varepsilon_{1}\right) e^{d_{M} s} d s\right]
\end{gathered}
$$

$$
\left.\times e^{-d_{M} T}+\delta_{M}\right)\left(1-\left(1-P_{M}\right) e^{-d_{M} T}\right)^{-1},
$$

$\widetilde{y_{M}^{*}\left(\tau T^{+}\right)}$

$$
\begin{aligned}
& =\left(\left(1-P_{M}\right)\right. \\
& \times\left[m \int_{0}^{\tau T}\left(\widetilde{y_{J}^{*}(t)}+\varepsilon_{1}\right) e^{d_{M} s} d s\right. \\
& \left.\quad+m e^{-d_{M} T} \int_{\tau T}^{T}\left(\widetilde{y_{J}^{*}(t)}+\varepsilon_{1}\right) e^{d_{M} s} d s+\delta_{M}\right] \\
& \left.\times e^{-d_{M} \tau T}\right)\left(1-\left(1-P_{M}\right) e^{-d_{M} T}\right)^{-1} .
\end{aligned}
$$

We shall prove that one cannot have $S(t)<m_{5}$ for all $t>0$, otherwise

$$
\begin{aligned}
& x^{\prime}(t) \geq\left(r-P_{1}^{\prime}(0) m_{5}\right) x(t)\left(1-\frac{r x(t)}{K\left(r-P_{1}^{\prime}(0) m_{5}\right)}\right), \\
& I^{\prime}(t) \leq-\left(d_{I}-g^{\prime}(0) m_{5}\right) I(t), \\
& y_{J}^{\prime}(t) \leq \lambda P_{2}\left(m_{5}\right) M-\left(d_{J}+m\right) y_{J}(t), \\
& y_{M}^{\prime}(t)=m y_{J}(t)-d_{M} y_{M}(t), \\
& t \neq(n+\tau-1) T, \\
& t \neq n T, \\
& \Delta x(t)=-\delta x(t), \\
& \Delta I(t)=-P_{I} I(t), \\
& \Delta y_{J}(t)=-P_{J} y_{J}(t) \text {, } \\
& \Delta y_{M}(t)=-P_{M} y_{M}(t) \text {, } \\
& t=(n+\tau-1) T, \\
& \Delta x(t)=0, \\
& \Delta I(t)=\delta_{I}, \\
& \Delta y_{J}(t)=\delta_{J}, \\
& \Delta y_{M}(t)=\delta_{M} \text {, } \\
& t=n T \text {. }
\end{aligned}
$$

Analyzing (61) with similarity as (40), it is easy to obtain that there exists a positive constant $n_{6}^{*}$, such that $x(t) \geq \widetilde{x^{*}(t)}-$ $\varepsilon_{1}, I(t) \leq \widetilde{I^{*}(t)}+\varepsilon_{1}, y_{J}(t) \leq \widetilde{y_{J}^{*}(t)}+\varepsilon_{1}, y_{M}(t) \leq \widetilde{y_{M}^{*}(t)}+\varepsilon_{1}$ for $t \geq n_{6}^{*} T$. Therefore,

$$
\begin{aligned}
S^{\prime}(t) \geq & \beta P_{1}\left(\widetilde{x^{*}(t)}-\varepsilon_{1}\right) S(t)-g\left(\widetilde{I^{*}(t)}+\varepsilon_{1}\right) S(t) \\
& -P_{2}^{\prime}(0)\left(\widetilde{y_{M}^{*}(t)}+\varepsilon_{1}\right) S(t)-d_{S} S(t),
\end{aligned}
$$




$$
\begin{gathered}
t \neq(n+\tau-1) T, \quad t \neq n T, \\
\Delta S(t)=-P_{S} S(t), \quad t=(n+\tau-1) T, \\
\Delta S(t)=0, \quad t=n T,
\end{gathered}
$$

for $t \geq n_{6}^{*} T$. Let $N_{0} \in \mathbb{N}$ and $\left(N_{0}+\tau-1\right) \geq n_{6}^{*}$. Integrating (62) on $((n+\tau-1) T,(n+\tau) T], n \geq N_{0}$, we have

$$
\begin{aligned}
S((n+\tau) T) \\
\geq S((n+\tau-1) T)\left(1-P_{S}\right) \\
\quad \times e^{\int_{(n+\tau-1) T}^{(n+\tau) T}\left(\beta P_{1}\left(\widetilde{x^{*}(t)}-\varepsilon_{1}\right)-g\left(\widetilde{I^{*}(t)}+\varepsilon_{1}\right)-P_{2}^{\prime}(0)\left(\overline{y_{M}^{*}(t)}+\varepsilon_{1}\right)-d_{S}\right) d t} \\
=S((n+\tau-1) T) e^{\eta} .
\end{aligned}
$$

Then $S\left(\left(N_{0}+\tau+k\right) T\right) \geq S\left(\left(N_{0}+\tau\right) T\right) e^{k \eta} \rightarrow \infty$ as $k \rightarrow \infty$, which is a contradiction. So there exists a $t_{1}\left(t_{1}>n_{6}^{*} T\right)$ such that $S\left(t_{1}\right) \geq m_{5}$.

Step 2. If $S(t) \geq m_{5}$ for all $t \geq t_{1}$, then Our purpose is obtained. If not, let $t_{2}=\inf \left\{t>t_{1} \mid S(t)<m_{5}\right\}$. Then $S(t) \geq m_{5}$ for $t \in\left[t_{1}, t_{2}\right)$ and $S\left(t_{2}\right)=m_{5}$. In this step, we consider two possible cases for $t_{2}$.

Case 1. $t_{2}=\left(n_{1}+\tau-1\right) T, n_{1} \in \mathbb{N}$. Then $S\left(t_{2}^{+}\right)=(1-$ $\left.P_{S}\right) S\left(t_{2}\right)<m_{5}$. Select $n_{2}, n_{3} \in \mathbb{N}$ such that $\left(n_{2}-1\right) \geq n_{6}^{*}$ and $\left(1-P_{S}\right)^{n_{2}} e^{n_{3} \eta+n_{2} \sigma T}>\left(1-P_{S}\right)^{n_{2}} e^{n_{3} \eta+\left(n_{2}+1\right) \sigma T}>1$, where $\sigma=\beta P_{1}\left(m_{4}\right)-g(M)-P_{2}^{\prime}(0) M-d_{S}<0$. Let $\widetilde{T}=\left(n_{2}+n_{3}\right) T$, then we have the claim: there exists $t_{3} \in\left(t_{2}, t_{2}+\widetilde{T}\right]$ such that $S\left(t_{3}\right) \geq m_{5}$. If the claim is false, we will get a contradiction in the following.

According to Step 1, we have $x(t) \geq \widetilde{x^{*}(t)}-\varepsilon_{1}, I(t) \leq$ $\widetilde{I^{*}(t)}+\varepsilon_{1}, y_{J}(t) \leq \widetilde{y_{J}^{*}(t)}+\varepsilon_{1}, y_{M}(t) \leq \widetilde{y_{M}^{*}(t)}+\varepsilon_{1}$ for $t \geq$ $\left(n_{1}+n_{2}-1\right) T$. Then, we have

$$
\begin{gathered}
S^{\prime}(t) \geq \beta P_{1}\left(\widetilde{x^{*}(t)}-\varepsilon_{1}\right) S(t)-g\left(\widetilde{I^{*}(t)}+\varepsilon_{1}\right) S(t) \\
-P_{2}^{\prime}(0)\left(\widetilde{y_{M}^{*}(t)}+\varepsilon_{1}\right) S(t)-d_{S} S(t), \\
t \neq(n+\tau-1) T, \quad t \neq n T, \\
\Delta S(t)=-P_{S} S(t), \quad t=(n+\tau-1) T, \\
\Delta S(t)=0, \quad t=n T,
\end{gathered}
$$

for $t \in\left[t_{2}+n_{2} T, t_{2}+\widetilde{T}\right]$. As in Step 1, we have

$$
S\left(t_{2}+\widetilde{T}\right) \geq S\left(t_{2}+n_{2} T\right) e^{n_{3} \eta} .
$$

Since $x(t) \geq m_{4}, I(t) \leq M, y_{M}(t) \leq M$ and $P_{2}(S(t))<$ $P_{2}^{\prime}(0) S(t)$, we have

$$
\begin{array}{r}
S^{\prime}(t) \\
\geq\left(\beta P_{1}\left(m_{4}\right)-g(M)-P_{2}^{\prime}(0) M-d_{S}\right) S(t)=\sigma S(t), \\
t \neq(n+\tau-1) T, \quad t \neq n T, \\
\Delta S(t)=-P_{S} S(t), \quad t=(n+\tau-1) T, \\
\Delta S(t)=0, \quad t=n T,
\end{array}
$$

for $t \in\left[t_{2}, t_{2}+n_{2} T\right]$. Integrating (66) on $\left[t_{2}, t_{2}+n_{2} T\right]$, we have

$$
\begin{aligned}
S\left(t_{2}+n_{2} T\right) & \geq S\left(t_{2}^{+}\right) e^{n_{2} \sigma T} \\
& =\left(1-P_{S}\right) m_{5} e^{n_{2} \sigma T} \\
& \geq\left(1-P_{S}\right)^{n_{2}} m_{5} e^{n_{2} \sigma T} .
\end{aligned}
$$

Thus, by (65) and (67), we have $S\left(t_{2}+\widetilde{T}\right) \geq(1-$ $\left.P_{S}\right)^{n_{2}} m_{5} e^{n_{3} \eta+n_{2} \sigma T}>m_{5}$, which is a contradiction. Let $t_{4}=$ $\inf \left\{t>t_{2} \mid S(t) \geq m_{5}\right\}$, then for $t \in\left[t_{2}, t_{4}\right), S(t)<m_{5}$ and $S\left(t_{4}\right)=m_{5}$. So, $S(t) \geq S\left(t_{2}^{+}\right) e^{\sigma\left(t-t_{2}\right)}=\left(1-P_{S}\right) m_{5} e^{\sigma\left(t-t_{2}\right)} \geq$ $\left(1-P_{S}\right)^{n_{2}+n_{3}} m_{5} e^{\sigma\left(n_{2}+n_{3}\right) T}=\widetilde{m_{1}}$ for $t \in\left[t_{2}, t_{4}\right)$.

Case $2\left(t_{2} \neq\left(n_{1}+\tau-1\right) T, n_{1} \in \mathbb{N}\right)$. Suppose that $t_{2} \in\left(\left(n_{1}^{\prime}+\right.\right.$ $\left.\tau-1) T,\left(n_{1}^{\prime}+\tau\right) T\right), n_{1}^{\prime} \in \mathbb{N}$. $S(t) \geq m_{5}$ for $t \in\left[t_{1}, t_{2}\right)$ and $S\left(t_{2}\right)=m_{5}$. There are two possible cases for $t \in\left(t_{2},\left(n_{1}^{\prime}+\tau\right) T\right)$.

Case $2 a$. If $S(t) \leq m_{5}$ for all $t \in\left(t_{2},\left(n_{1}^{\prime}+\tau\right) T\right)$, similar to Case 1 , we can prove there exists a $t_{3}^{\prime} \in\left(\left(n_{1}^{\prime}+\tau\right) T,\left(n_{1}^{\prime}+\tau\right) T+\widetilde{T}\right]$ such that $S\left(t_{3}^{\prime}\right) \geq m_{5}$. Let $t_{4}^{\prime}=\inf \left\{t>t_{2} \mid S(t) \geq m_{5}\right\}$, then for $t \in\left[t_{2}, t_{4}^{\prime}\right), S(t)<m_{5}$ and $S\left(t_{4}^{\prime}\right)=m_{5}$. So, $S(t) \geq S\left(t_{2}\right) e^{\sigma\left(t-t_{2}\right)}=$ $m_{5} e^{\sigma\left(t-t_{2}\right)} \geq\left(1-P_{S}\right)^{n_{2}+n_{3}} m_{5} e^{\sigma\left(n_{2}+n_{3}+1\right) T}=m^{*}<\widetilde{m_{1}}$ for all $t \in\left[t_{2}, t_{4}^{\prime}\right)$.

Case $2 b$. If there exists a $t \in\left(t_{2},\left(n_{1}^{\prime}+\tau\right) T\right)$ such that $S(t) \geq m_{5}$. Let $\overline{t_{4}^{\prime}}=\inf \left\{t>t_{2} \mid S(t) \geq m_{5}\right\}$, then for $t \in\left[t_{2}, \overline{t_{4}^{\prime}}\right), S(t)<$ $m_{5}$ and $S\left(\overline{t_{4}^{\prime}}\right)=m_{5}$. So, $S(t) \geq S\left(t_{2}\right) e^{\sigma\left(t-t_{2}\right)}=m_{5} e^{\sigma\left(t-t_{2}\right)} \geq$ $m_{5} e^{\sigma T}>m^{*}$ for all $t \in\left[t_{2}, \overline{t_{4}^{\prime}}\right)$.

Since $S(t) \geq m_{5}$ for some $t \geq t_{1}$, in both cases a similar discussion can be continued. The proof is completed.

\section{Numerical Simulations and Conclusions}

In this section, we will give an example and its simulations to show the efficiency of the criteria derived in Section 4.

In system (1), let $P_{1}(x(t))=a x(t), g(I(t))=b I(t)$, and $P_{2}(S(t))=h\left(1-e^{-c S(t)}\right), a, b, c, h>0$. Namely, $P_{1}(x(t))$ describes an Holling type-I functional response of the pest, $P_{2}(S(t))$ describes a Ivlev-type functional response of the pest's natural predator. Therefore, we consider the pest management model with impulsive releasing and harvesting at two different fixed moments:

$$
\begin{aligned}
x^{\prime}(t)= & r x(t)\left(1-\frac{x(t)}{K}\right)-a x(t) S(t), \\
S^{\prime}(t)= & \beta a x(t) S(t)-b I(t) S(t) \\
& -h\left(1-e^{-c S(t)}\right) y_{M}(t)-d_{S} S(t), \\
I^{\prime}(t)= & b I(t) S(t)-d_{I} I(t), \\
y_{J}^{\prime}(t)= & \lambda h\left(1-e^{-c S(t)}\right) y_{M}(t)-d_{J} y_{J}(t)-m y_{J}(t), \\
y_{M}^{\prime}(t)= & m y_{J}(t)-d_{M} y_{M}(t),
\end{aligned}
$$

$t \neq(n+\tau-1) T$,

$t \neq n T$, 


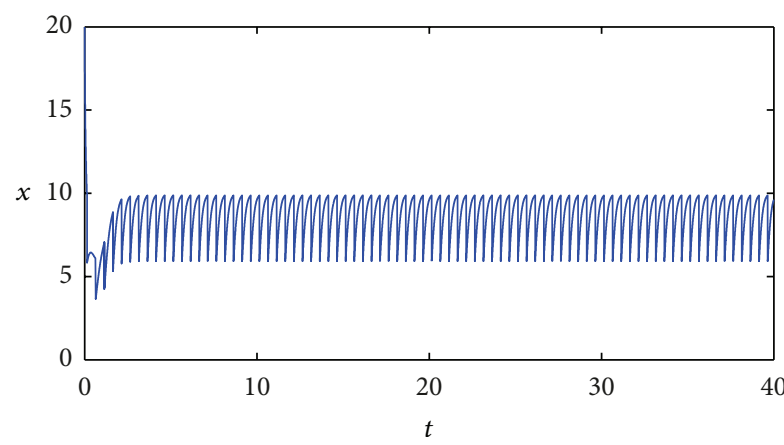

(a)

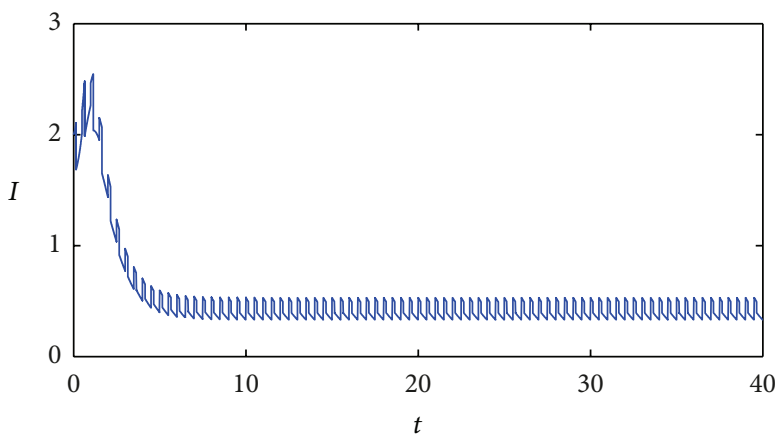

(c)

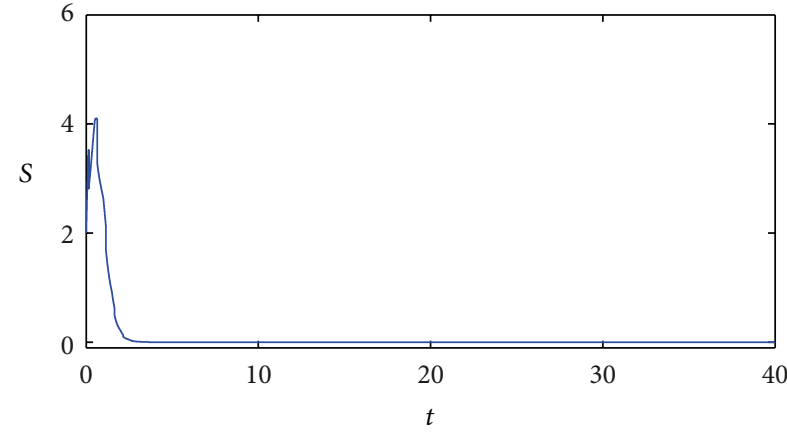

(b)

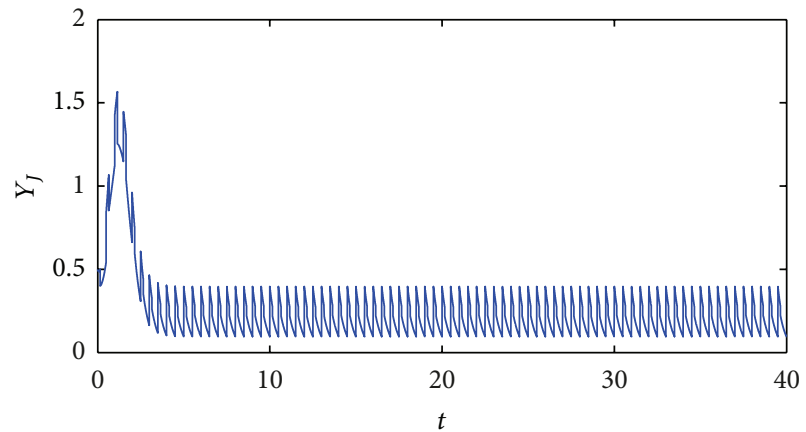

(d)

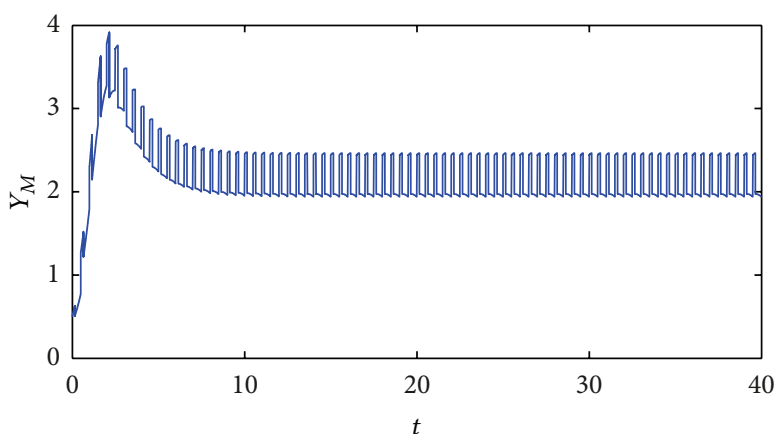

(e)

Figure 1: Time series of the system (68) with $r=8, K=10, a=0.8, \beta=0.5, b=0.3, h=8, c=0.2, \lambda=0.6, m=2, d_{S}=0.2, d_{I}=0.5$, $d_{J}=0.4, d_{M}=0.2, \delta=0.4, P_{S}=P_{I}=P_{J}=P_{M}=0.2, \delta_{I}=0.2, \delta_{J}=0.3, \delta_{M}=0.5, \tau=0.3, T=0.5, x(0)=20, S(0)=2, I(0)=2, y_{J}(0)=0.5$, $y_{M}(0)=0.5$.

$$
\begin{aligned}
& \Delta x(t)=-\delta x(t), \\
& \Delta S(t)=-P_{S} S(t), \\
& \Delta I(t)=-P_{I} I(t), \\
& \Delta y_{J}(t)=-P_{J} y_{J}(t), \\
& \Delta y_{M}(t)=-P_{M} y_{M}(t), \\
& \quad t=(n+\tau-1) T, \\
& \Delta x(t)=0, \\
& \Delta S(t)=0, \\
& \Delta I(t)=\delta_{I}, \\
& \Delta y_{J}(t)=\delta_{J},
\end{aligned}
$$

$$
\begin{array}{r}
\Delta y_{M}(t)=\delta_{M}, \\
t=n T .
\end{array}
$$

So, by (22), (23), and (25), we have

$$
\begin{aligned}
& \beta \int_{0}^{T} P_{1}\left(x^{*}(t)\right) d t=\frac{\beta a K}{r}(r T+\ln (1-\delta))=\theta_{1}, \\
& \int_{0}^{T} g\left(I^{*}(t)\right) d t \\
& \quad=\frac{b \delta_{I}\left[1-e^{-d_{I} \tau T}+\left(1-P_{I}\right)\left(e^{-d_{I} \tau T}-e^{-d_{I} T}\right)\right]}{d_{I}\left(1-\left(1-P_{I}\right) e^{-d_{I} T}\right)} \\
& \quad=\theta_{2},
\end{aligned}
$$




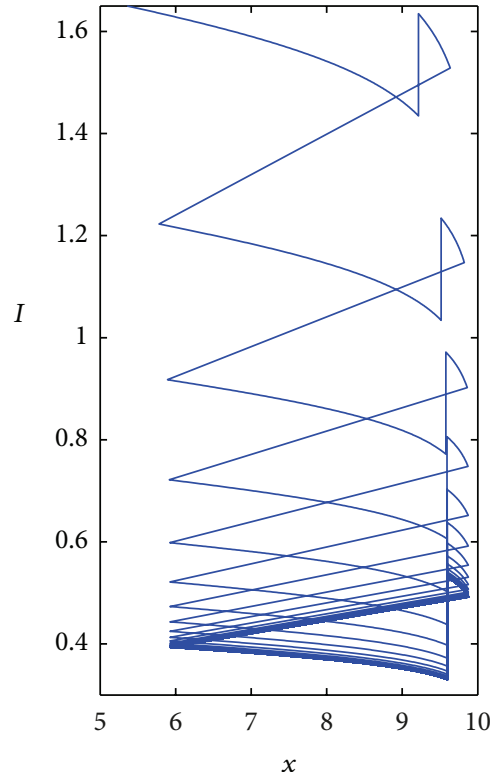

(a)

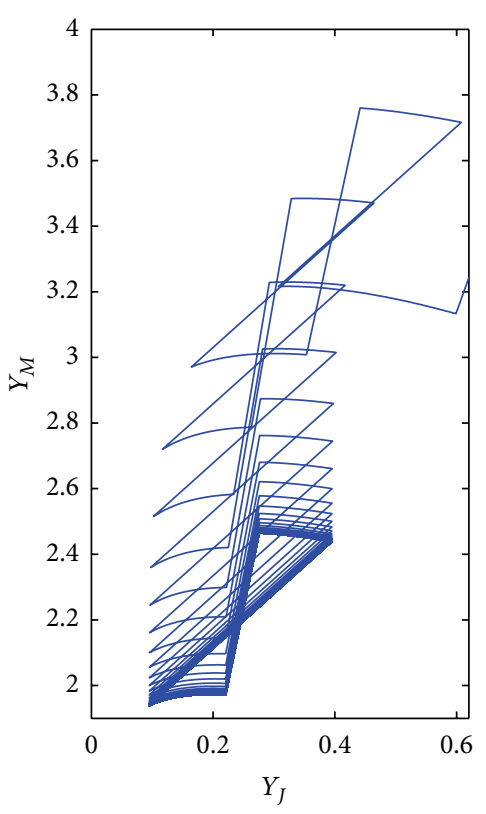

(b)

FIGURE 2: Phase portrait of the system (68) with $r=8, K=10, a=0.8, \beta=0.5, b=0.3, h=8, c=0.2, \lambda=0.6, m=2, d_{S}=0.2, d_{I}=0.5$, $d_{J}=0.4, d_{M}=0.2, \delta=0.4, P_{S}=P_{I}=P_{J}=P_{M}=0.2, \delta_{I}=0.2, \delta_{J}=0.3, \delta_{M}=0.5, \tau=0.3, T=0.5, x(0)=20, S(0)=2, I(0)=2, y_{J}(0)=0.5$, $y_{M}(0)=0.5$.

$$
\begin{aligned}
& \int_{0}^{T} y_{M}^{*}(t) d t \\
& =\frac{y_{M}^{*}\left(0^{+}\right)}{d_{M}}\left(1-e^{-d_{M} \tau T}\right) \\
& +\frac{m \delta_{J}}{\left(1-\left(1-P_{J}\right) e^{-\left(m+d_{J}\right) T}\right)\left(d_{M}-\left(m+d_{J}\right)\right)} \\
& \quad \times\left(\frac{1-e^{-\left(m+d_{J}\right) \tau T}}{m+d_{J}}-\frac{1-e^{-d_{M} \tau T}}{d_{M}}\right) \\
& +\frac{y_{M}^{*}\left(\tau T^{+}\right) e^{d_{M} \tau T}}{d_{M}}\left(e^{-d_{M} \tau T}-e^{d_{M} T}\right) \\
& +\theta_{3}, \\
& \quad \times\left(\frac{e^{-\left(m+d_{J}\right) \tau T}-e^{-\left(m+d_{J}\right) T}}{m+d_{J}}\left(1-P_{J}\right)\right. \\
& +\left(1-\left(1-P_{J}\right) e^{-\left(m+d_{J}\right) T}\right)\left(d_{M}-\left(m+d_{J}\right)\right)
\end{aligned}
$$

where

$$
\begin{gathered}
y_{M}^{*}\left(0^{+}\right)=\frac{\left[\left(1-P_{M}\right) A(\tau T)+B(T)\right] e^{-d_{M} T}+\delta_{M}}{1-\left(1-P_{M}\right) e^{-d_{M} T}}, \\
y_{M}^{*}\left(\tau T^{+}\right)=\frac{\left(1-P_{M}\right)\left[A(\tau T)+e^{-d_{M} T} B(T)+\delta_{M}\right] e^{-d_{M} \tau T}}{1-\left(1-P_{M}\right) e^{-d_{M} T}}, \\
A(\tau T)=\frac{m \delta_{J}\left(e^{\left(d_{M}-\left(m+d_{J}\right)\right) \tau T}-1\right)}{\left(1-\left(1-P_{J}\right) e^{-\left(m+d_{J}\right) T}\right)\left(d_{M}-\left(m+d_{J}\right)\right)}, \\
B(T)=\frac{m \delta_{J}\left(1-P_{J}\right)\left(e^{\left(d_{M}-\left(m+d_{J}\right)\right) T}-e^{\left(d_{M}-\left(m+d_{J}\right)\right) \tau T}\right)}{\left(1-\left(1-P_{J}\right) e^{-\left(m+d_{J}\right) T}\right)\left(d_{M}-\left(m+d_{J}\right)\right)} .
\end{gathered}
$$

Then, by Theorems 11 and 14, we have the following.

(T1) If $\theta_{1}-\theta_{2}-\theta_{3}-d_{S} T<\ln \left(1 /\left(1-P_{S}\right)\right)$, then the susceptible pest-eradication periodic solution $\left(x^{*}(t)\right.$, $\left.0, I^{*}(t), y_{J}^{*}(t), y_{M}^{*}(t)\right)$ of system (68) is locally asymptotically stable.

(T2) If $\theta_{1}-\theta_{2}-h c e^{-c M} \theta_{3}-d_{S} T<\ln \left(1 /\left(1-P_{S}\right)\right)$, then the susceptible pest-eradication periodic solution $\left(x^{*}(t), 0, I^{*}(t), y_{J}^{*}(t), y_{M}^{*}(t)\right)$ of system (68) is globally asymptotically stable, where $M$ is defined in Lemma 6.

(T3) If $\theta_{1}-\theta_{2}-h c \theta_{3}-d_{S} T>\ln \left(1 /\left(1-P_{S}\right)\right.$, then system (68) is permanent.

In the following, we analyze the locally asymptotical stability of the susceptible pest-eradication periodic solution and permanence of system (68). 


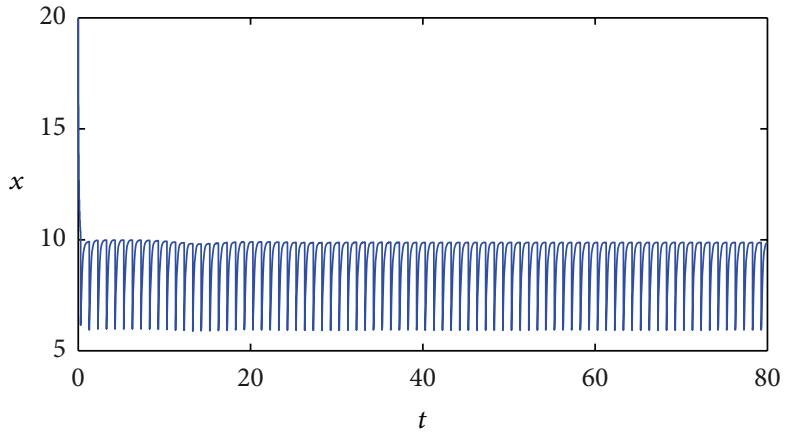

(a)

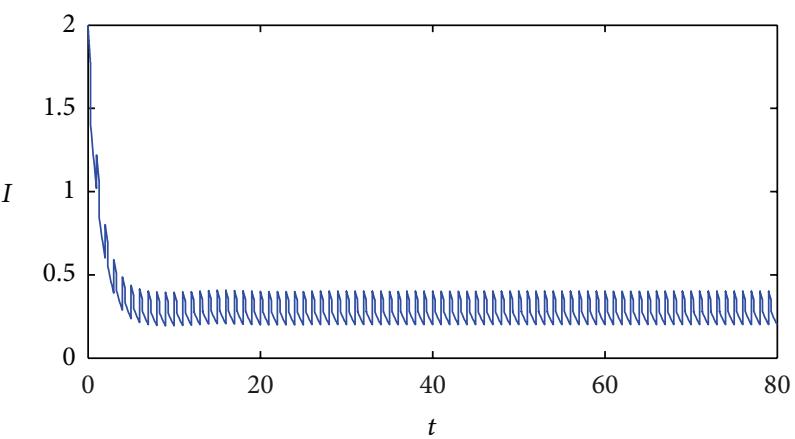

(c)

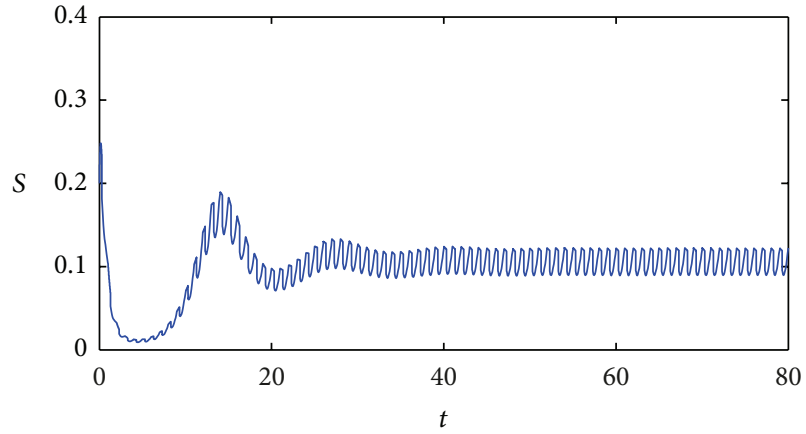

(b)

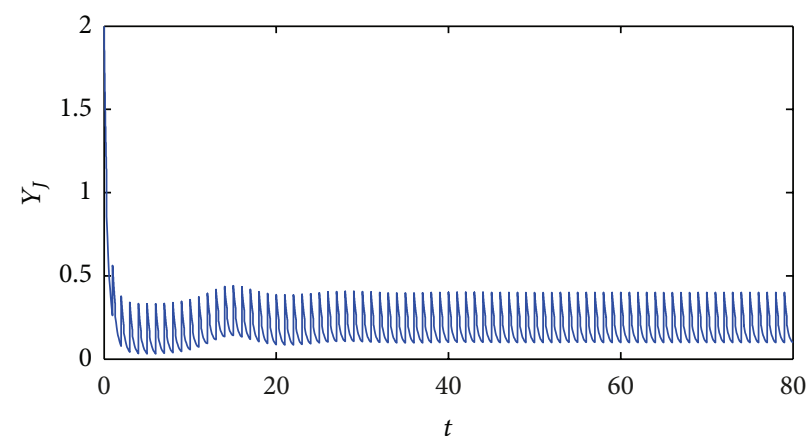

(d)

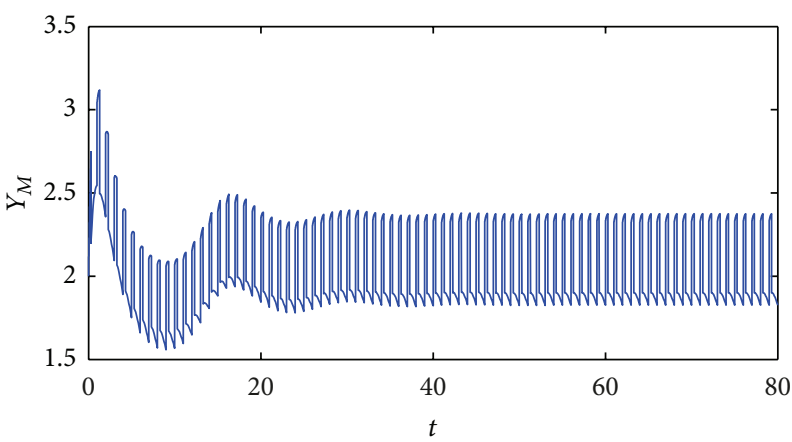

(e)

FIGURE 3: Time series of the system (68) with $r=8, K=10, a=0.8, \beta=0.5, b=0.3, h=8, c=0.2, \lambda=0.6, m=2, d_{S}=0.2, d_{I}=0.5$, $d_{J}=0.4, d_{M}=0.2, \delta=0.4, P_{S}=P_{I}=P_{J}=P_{M}=0.2, \delta_{I}=0.2, \delta_{J}=0.3, \delta_{M}=0.5, \tau=0.3, T=1, x(0)=20, S(0)=0.2, I(0)=2, y_{J}(0)=2$, $y_{M}(0)=2$.

Assume that $x(0)=20, S(0)=2, I(0)=2, y_{I}(0)=0.5$, $y_{M}(0)=0.5, r=8, K=10, a=0.8, \beta=0.5, b=0.3, h=8$, $c=0.2, \lambda=0.6, m=2, d_{S}=0.2, d_{I}=0.5, d_{I}=0.4, d_{M}=0.2$, $\delta=0.4, P_{S}=P_{I}=P_{J}=P_{M}=0.2, \delta_{I}=0.2, \delta_{J}=0.3$, $\delta_{M}=0.5, \tau=0.3, T=0.5$. Obviously, the condition of $(T 1)$ is satisfied, then the susceptible pest-eradication periodic solution of system (68) is locally asymptotically stable, which can be seen from the numerical simulation in Figures 1 and 2.

Assume that $x(0)=20, S(0)=0.2, I(0)=2, y_{J}(0)=2$, $y_{M}(0)=2, r=8, K=10, a=0.8, \beta=0.5, b=0.3, h=8$, $c=0.2, \lambda=0.6, m=2, d_{S}=0.2, d_{I}=0.5, d_{J}=0.4, d_{M}=0.2$, $\delta=0.4, P_{S}=P_{I}=P_{J}=P_{M}=0.2, \delta_{I}=0.2, \delta_{J}=0.3$, $\delta_{M}=0.5, \tau=0.3, T=1$. Obviously, the condition of (T3) is satisfied. Then, system (68) is permanent, which can also be seen from Figures 3 and 4.

From results of the numerical simulation, we know that there exists an impulsive harvesting(or releasing) periodic threshold $T^{*}$, which satisfies $0.5<T^{*}<1$. If $T<T^{*}$ and the other parameters are fixed $(r=8, K=10, a=0.8$, $\beta=0.5, b=0.3, h=8, c=0.2, \lambda=0.6, m=2$, $d_{S}=0.2, d_{I}=0.5, d_{J}=0.4, d_{M}=0.2, \delta=0.4, P_{S}=$ $P_{I}=P_{J}=P_{M}=0.2, \delta_{I}=0.2, \delta_{J}=0.3, \delta_{M}=0.5, \tau=$ $0.3, T=0.5$.), then the susceptible pest-eradication periodic solution $\left(x^{*}(t), 0, I^{*}(t), y_{J}^{*}(t), y_{M}^{*}(t)\right)$ of system (68) is locally asymptotically stable. If $T>T^{*}$ and the other parameters are fixed $(r=8, K=10, a=0.8, \beta=0.5, b=0.3, h=8, c=0.2$, $\lambda=0.6, m=2, d_{S}=0.2, d_{I}=0.5, d_{J}=0.4, d_{M}=0.2$, $\delta=0.4, P_{S}=P_{I}=P_{J}=P_{M}=0.2, \delta_{I}=0.2, \delta_{J}=0.3$, $\delta_{M}=0.5, \tau=0.3, T=0.5$.), then system (68) is permanent. The same discussion can be applied to other parameters.

In this paper, we proposed a pest management model with impulsive releasing (periodic infective pests, immature and mature natural enemies releasing) and harvesting (periodic crops harvesting) at two different fixed moments. By means 


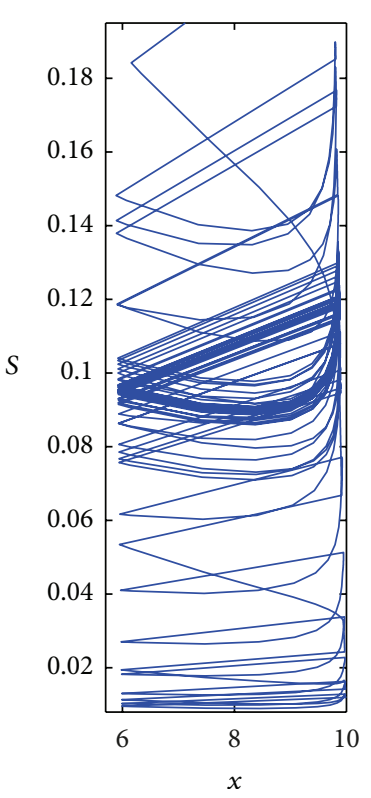

(a)

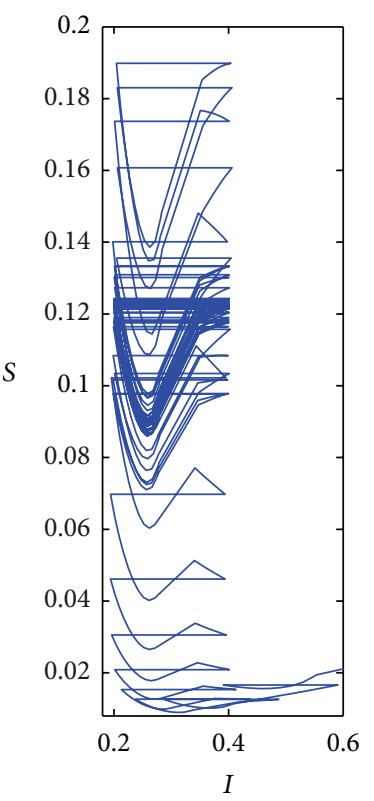

(b)

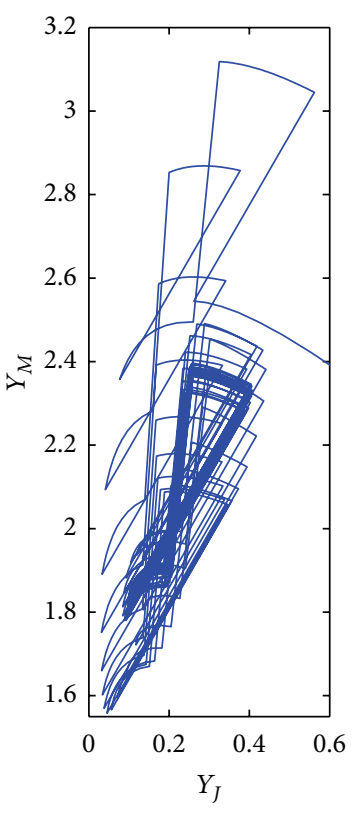

(c)

Figure 4: Phase portrait of the system (68) with $r=8, K=10, a=0.8, \beta=0.5, b=0.3, h=8, c=0.2, \lambda=0.6, m=2, d_{S}=0.2, d_{I}=0.5$, $d_{J}=0.4, d_{M}=0.2, \delta=0.4, P_{S}=P_{I}=P_{J}=P_{M}=0.2, \delta_{I}=0.2, \delta_{J}=0.3, \delta_{M}=0.5, \tau=0.3, T=1, x(0)=20, S(0)=0.2, I(0)=2, y_{J}(0)=2$, $y_{M}(0)=2$.

of Floquet theory and multicomparison results for impulsive differential equations, two sufficient conditions ensuring the locally and globally asymptotical stability of the susceptible pest-eradication period solution and permanence of the system are derived.

\section{Acknowledgments}

This work is supported partially by National Natural Science Foundation of China under Grant no. 10971240 and no. 61004042, Key Project of Chinese Education Ministry under Grant no. 212138, Natural Science Foundation of Chongqing under Grant CQ CSTC 2011BB0117, and Foundation of Science and Technology Project of Chongqing Education Commission under Grant KJ120630.

\section{References}

[1] P. Georgescu and G. Moroşanu, "Pest regulation by means of impulsive controls," Applied Mathematics and Computation, vol. 190, no. 1, pp. 790-803, 2007.

[2] T. Zhang, X. Meng, and Y. Song, "The dynamics of a highdimensional delayed pest management model with impulsive pesticide input and harvesting prey at different fixed moments," Nonlinear Dynamics, vol. 64, no. 1-2, pp. 1-12, 2011.

[3] Y. Ma, B. Liu, and W. Feng, "Dynamics of a birth-pulse singlespecies model with restricted toxin input and pulse harvesting," Discrete Dynamics in Nature and Society, vol. 2010, Article ID 142534, 20 pages, 2010.

[4] S. Cai, "A stage-structured single species model with pulse input in a polluted environment," Nonlinear Dynamics, vol. 57, no. 3 , pp. 375-382, 2009.
[5] B. Liu, L. Chen, and Y. Zhang, "The dynamics of a preydependent consumption model concerning impulsive control strategy," Applied Mathematics and Computation, vol. 169, no. 1, pp. 305-320, 2005.

[6] P. Georgescu, H. Zhang, and L. Chen, "Bifurcation of nontrivial periodic solutions for an impulsively controlled pest management model," Applied Mathematics and Computation, vol. 202, no. 2, pp. 675-687, 2008.

[7] P. Georgescu and H. Zhang, "An impulsively controlled predator-pest model with disease in the pest," Nonlinear Analysis. Real World Applications, vol. 11, no. 1, pp. 270-287, 2010.

[8] C. Wei and L. Chen, "Eco-epidemiology model with age structure and prey-dependent consumption for pest management," Applied Mathematical Modelling, vol. 33, no. 12, pp. 4354-4363, 2009.

[9] J. Jiao, S. Cai, and L. Chen, "Analysis of a stage-structured predatory-prey system with birth pulse and impulsive harvesting at different moments," Nonlinear Analysis. Real World Applications, vol. 12, no. 4, pp. 2232-2244, 2011.

[10] J. Jiao, G. Pang, L. Chen, and G. Luo, "A delayed stage-structured predator-prey model with impulsive stocking on prey and continuous harvesting on predator," Applied Mathematics and Computation, vol. 195, no. 1, pp. 316-325, 2008.

[11] Z. Liu and R. Tan, "Impulsive harvesting and stocking in a Monod-Haldane functional response predator-prey system," Chaos, Solitons and Fractals, vol. 34, no. 2, pp. 454-464, 2007.

[12] L. Nie, Z. Teng, L. Hu, and J. Peng, "The dynamics of a LotkaVolterra predator-prey model with state dependent impulsive harvest for predator," Biosystems, vol. 98, no. 2, pp. 67-72, 2009.

[13] D. Bănov and P. Simeonov, Impulsive Differential Equations: Periodic Solutions and Applications, John Wiley \& Sons, New York, NY, USA, 1993. 
[14] V. Lakshmikantham, D. D. Bainnov, and P. S. Simeonov, Theory of Impulsive Differential Equations, World Scientific, Singapore, 1989.

[15] T. Yang, Impulsive Control Theory, vol. 272, Springer, Berlin, Germany, 2001.

[16] C. S. Holling, "The functional response of predators to prey density and its role in mimicry and population regulation," Memoirs of the Entomological Society of Canada, vol. 45, pp. 560, 1965.

[17] Y. Wang and M. Zhao, "Dynamic analysis of an impulsively controlled predator-prey model with Holling type IV functional response," Discrete Dynamics in Nature and Society, vol. 2012, Article ID 141272, 18 pages, 2012.

[18] C. Shen, "Permanence and global attractivity of the foodchain system with Holling IV type functional response," Applied Mathematics and Computation, vol. 194, no. 1, pp. 179-185, 2007.

[19] X. Song and Y. Li, "Dynamic behaviors of the periodic predator-prey model with modified Leslie-Gower Holling-type II schemes and impulsive effect," Nonlinear Analysis. Real World Applications, vol. 9, no. 1, pp. 64-79, 2008.

[20] B. Liu, Z. Teng, and L. Chen, "Analysis of a predator-prey model with Holling II functional response concerning impulsive control strategy," Journal of Computational and Applied Mathematics, vol. 193, no. 1, pp. 347-362, 2006.

[21] B. Liu, Y. Zhang, and L. Chen, "Dynamic complexities of a Holling I predator-prey model concerning periodic biological and chemical control," Chaos, Solitons and Fractals, vol. 22, no. 1, pp. 123-134, 2004.

[22] X. Liu and L. Chen, "Complex dynamics of Holling type II Lotka-Volterra predator-prey system with impulsive perturbations on the predator," Chaos, Solitons and Fractals, vol. 16, no. 2, pp. 311-320, 2003.

[23] X. Song and Y. Li, "Dynamic complexities of a Holling II twoprey one-predator system with impulsive effect," Chaos, Solitons \& Fractals, vol. 33, no. 2, pp. 463-478, 2007.

[24] S. Zhang, D. Tan, and L. Chen, "Chaos in periodically forced Holling type IV predator-prey system with impulsive perturbations," Chaos, Solitons and Fractals, vol. 27, no. 4, pp. 980-990, 2006.

[25] S. Zhang, F. Wang, and L. Chen, "A food chain model with impulsive perturbations and Holling IV functional response," Chaos, Solitons and Fractals, vol. 26, no. 3, pp. 855-866, 2005.

[26] L.-L. Wang and W.-T. Li, "Periodic solutions and permanence for a delayed nonautonomous ratio-dependent predator-prey model with Holling type functional response," Journal of Computational and Applied Mathematics, vol. 162, no. 2, pp. 341-357, 2004.

[27] V. S. Ivlev, Experimental Ecology of the Feeding of Fishes, Yale University Press, New Haven, Conn, USA, 1961.

[28] H. K. Baek, S. D. Kim, and P. Kim, "Permanence and stability of an Ivlev-type predator-prey system with impulsive control strategies," Mathematical and Computer Modelling, vol. 50, no. 9-10, pp. 1385-1393, 2009.

[29] H. Wang and W. Wang, "The dynamical complexity of a Ivlev-type prey-predator system with impulsive effect," Chaos, Solitons \& Fractals, vol. 38, no. 4, pp. 1168-1176, 2008.

[30] Z. Xiang and X. Song, "The dynamical behaviors of a food chain model with impulsive effect and Ivlev functional response," Chaos, Solitons \& Fractals, vol. 39, no. 5, pp. 2282-2293, 2009.

[31] X. Song, M. Hao, and X. Meng, "A stage-structured predatorprey model with disturbing pulse and time delays," Applied Mathematical Modelling, vol. 33, no. 1, pp. 211-223, 2009. 


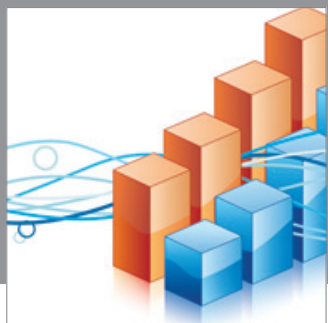

Advances in

Operations Research

mansans

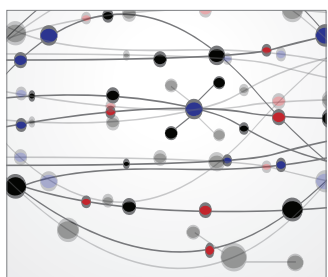

The Scientific World Journal
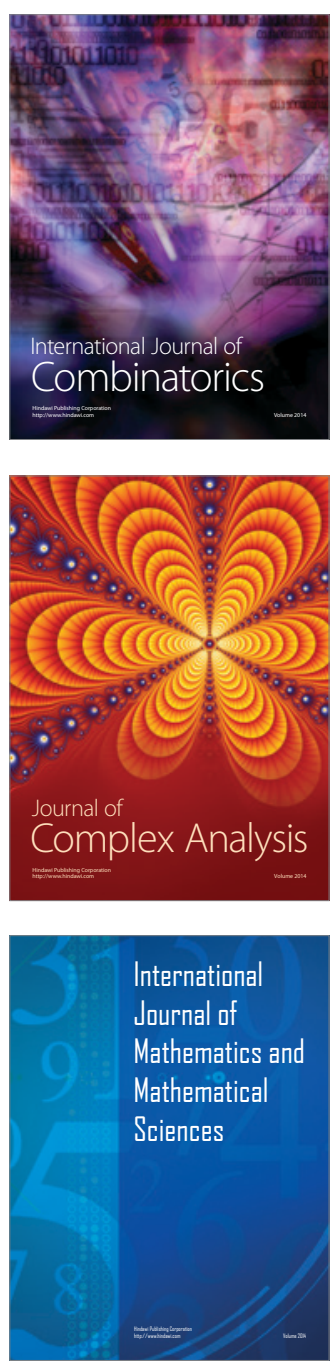
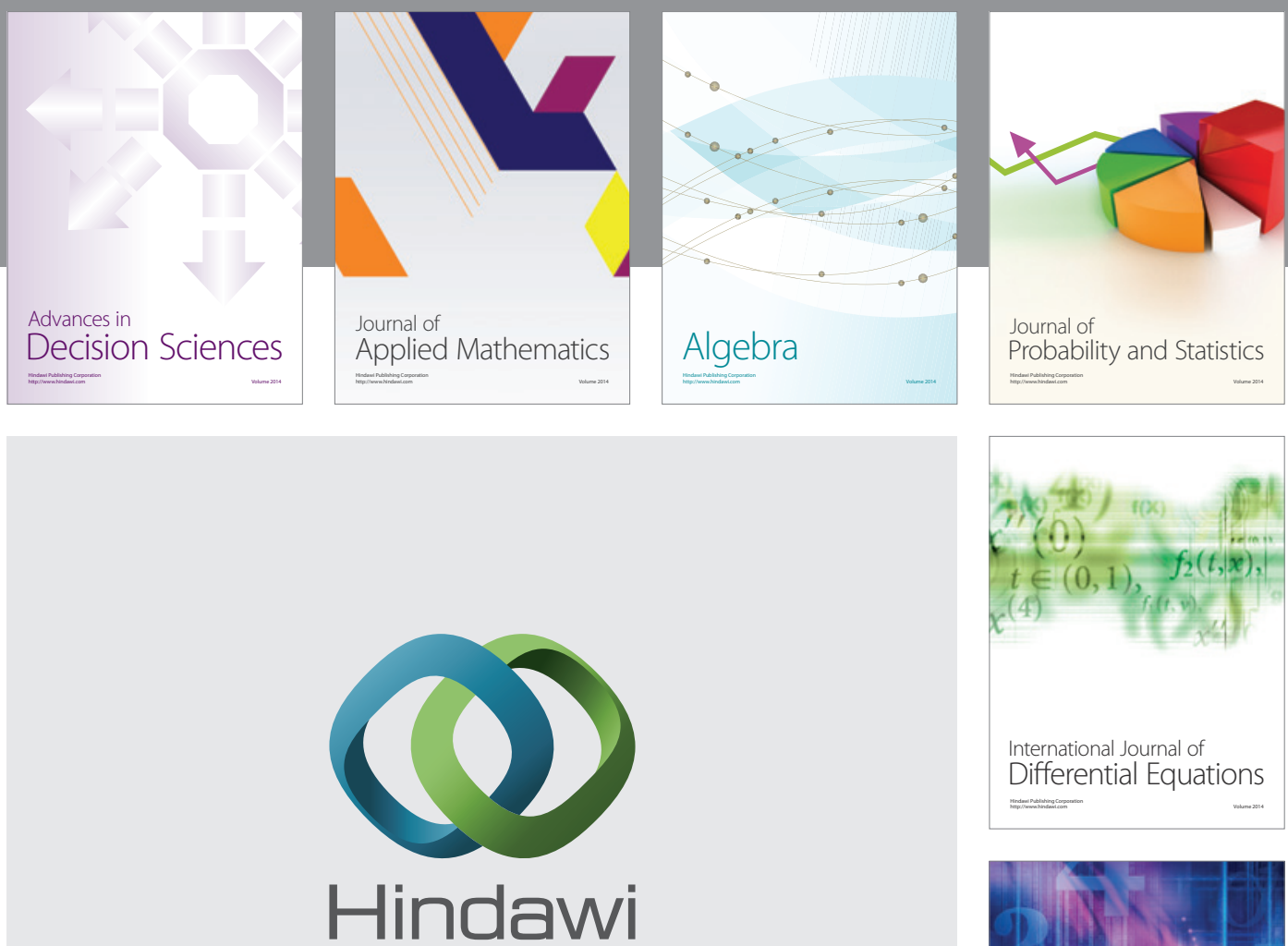

Submit your manuscripts at http://www.hindawi.com
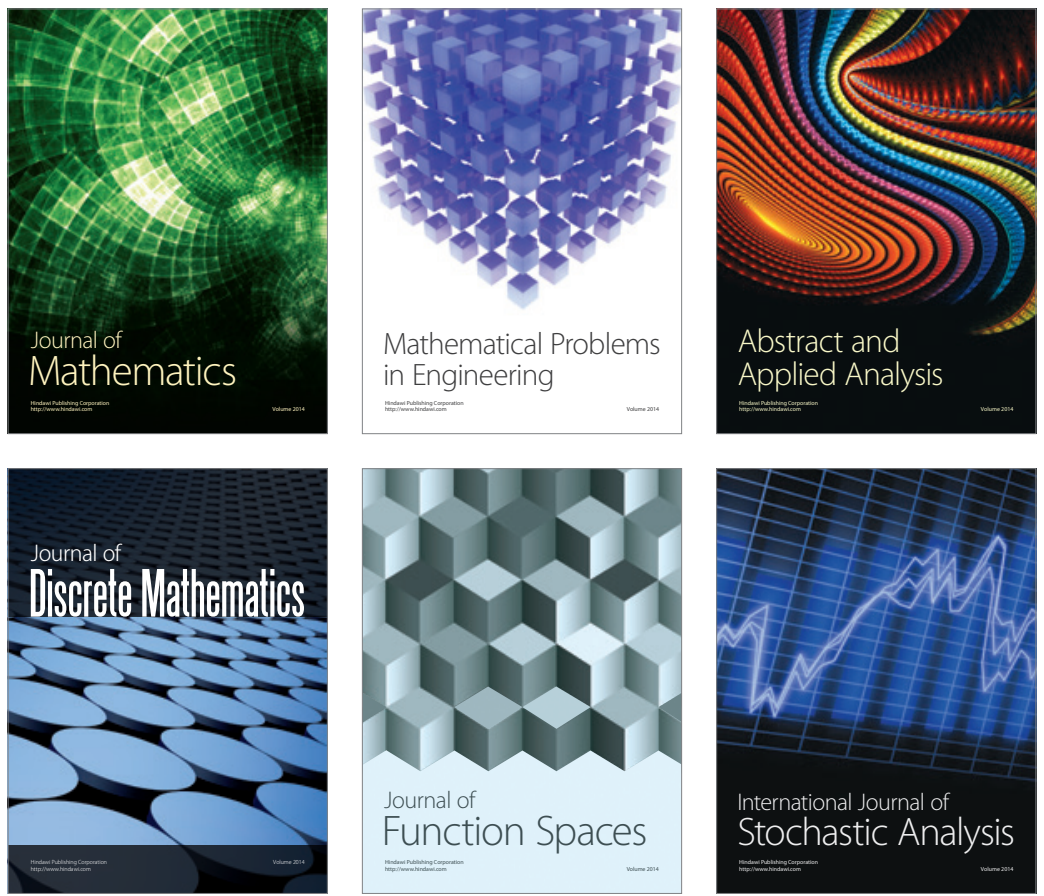

Journal of

Function Spaces

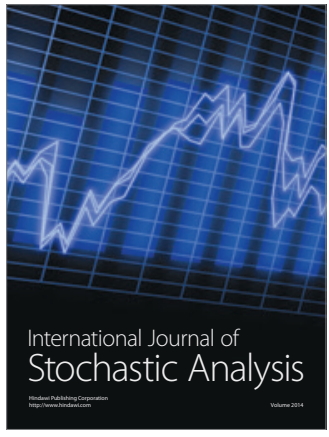

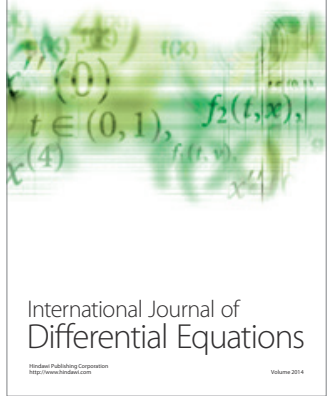
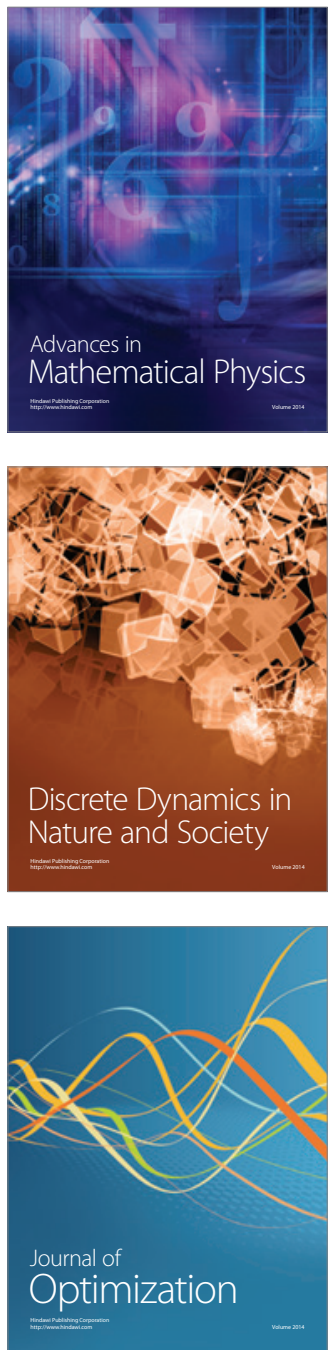